\author{
UNIVERSIDADE DE SÃO PAULO \\ Faculdade de Odontologia de Ribeirão Preto \\ Departamento de Clínica Infantil, Odontologia Preventiva e Social
}

LUCIANE ALMEIDA DO CARMO

“ÁREA DA NASOFARINGE APÓS A EXPANSÃo RÁPIDA DA MAXILA POR MEIO DE RADIOGRAFIA CEFALOMÉTRICA LATERAL E RESSONÂNCIA MAGNÉTICA"

Ribeirão Preto 
LUCIANE ALMEIDA DO CARMO

“ÁREA dA NASOFARINGE APÓS A EXPANSÃo RÁPIDA DA MAXILA POR MEIO
DE RADIOGRAFIA CEFALOMÉTRICA LATERAL E RESSONÂNCIA MAGNÉTICA"

Dissertação apresentada à Faculdade de Odontologia de Ribeirão Preto da Universidade de São Paulo para a obtenção do título de Mestre em Ciências. Área de Concentração: Odontopediatria.

Orientadora: Profa. Dra. Mírian Aiko Nakane Matsumoto

Ribeirão Preto 
AUTORIZO A REPRODUÇÃO E DIVULGAÇÃO TOTAL OU PARCIAL DESTE TRABALHO, POR QUALQUER MEIO CONVENCIONAL OU ELETRÔNICO, PARA FINS DE ESTUDO E PESQUISA, DESDE QUE CITADA A FONTE.

FICHA CATALOGRÁFICA

Carmo, Luciane Almeida

Área da nasofaringe após a expansão rápida da maxila por meio de radiografia cefalométrica lateral e ressonância magnética. Ribeirão Preto, 2011.

59 p. : il. ; $30 \mathrm{~cm}$

Dissertação de Mestrado, apresentada à Faculdade de Odontologia de Ribeirão Preto/USP. Área de concentração: Odontopediatria.

Orientador: Matsumoto, Mírian Aiko Nakane.

Palavras-chave: 1. Imagem por ressonância magnética. 2. Radiografia cefalométrica lateral. 3. Nasofaringe. 4. Técnica de expansão palatina 


\section{“ÁREA DA NASOFARINGE APÓS A EXPANSÃo RÁPIDA DA MAXILA POR MEIO DE RADIOGRAFIA CEFALOMÉTRICA LATERAL E RESSONÂNCIA MAGNÉTICA"}

Dissertação de Mestrado apresentada à Faculdade de Odontologia de Ribeirão Preto da Universidade de São Paulo para a obtenção do título de Mestre em Ciências. Área de concentração: Odontopediatria.

Data da defesa:

Banca Examinadora

$\operatorname{Prof}(a) . \operatorname{Dr}(\mathrm{a}) .:$

Instituição:

Julgamento:

Assinatura:

Prof(a). Dr(a).:

Instituição:

Julgamento: Assinatura:

Prof(a). Dr(a).:

Instituição:

Julgamento: Assinatura: 


\section{DADOS CURRICULARES}

\section{LUCIANE ALMEIDA DO CARMO}

Nascimento

Filiação

$2000-2004$

$2009-2011$
10 de abril de 1982 - Ribeirão Preto, SP, Brasil

Edelcio Luiz Ferreira do Carmo

Eliane Maria Almeida do Carmo

Curso de Graduação

Faculdade de Odontologia de Piracicaba

Universidade Estadual de Campinas (UNICAMP)

Curso de Pós-Graduação (Mestrado) em Ciências

Área de concentração: Odontopediatria

Faculdade de Odontologia de Ribeirão Preto / USP 


\section{MEUS AGRADECIMMENTOS}

À Deus primeiramente, por ter me dado uma vida cheia de luz e esperança, por me acompanhar e guiar em todos os meus caminhos e me ajudar a superar os obstáculos e a ser perseverante.

À minha família, em especial meus avós maternos (Ignez e Maurício) por todo amor, carinho e apoio que sempre me deram e pelo exemplo de simplicidade e sabedoria.

À minha mãe (Eliane) pelo amor incondicional, incentivo, dedicação e amizade e pela fé em mim depositada em todos os meus projetos.

Ao meu irmão (Rafael) pelo exemplo de perseverança, força e coragem e por me mostrar que nossos sonhos não são impossíveis e que com atitude positiva e trabalho alcançamos nossos objetivos.

À minha orientadora Profa Dra Mírian Aiko Nakane Matsumoto, exemplo de disposição e garra. Sou grata pelos ensinamentos, incentivos, compreensão, confiança e amizade compartilhada. Obrigada pela oportunidade de ser sua orientada e percorrer essa etapa da minha vida.

À Profa Dra Wilma Anselmo Terezinha pelo exemplo de dedicação e amor à profissão. Obrigada por acreditar em mim e me incentivar a fazer o curso de mestrado, pelo incentivo, pelos ensinamentos e pela oportunidade de fazer parte e aprender com sua equipe.

À Profa Dra Fabiana Cardoso Valera, pelos ensinamentos, disposição em ajudar e pela colaboração valiosa em meus trabalhos.

À Profa Dra Kranya Victoria Diaz Serrano pelo incentivo, pelas palavras de apoio e carinho, pelos ensinamentos e constante suporte.

Ao Prof. Dr. Paulo Nelson Fifho, um mestre exemplar. Obrigada pela disponibilidade, dedicação, estímulo e paciência em todos os momentos. A sua forma de ensinar, aulas perfeitas, atuais e postura calma e educada sempre será um exemplo a ser seguido.

À Profa. Dra. Alexandra Mussolino de Queiroz pela forma atenciosa, alegre e amiga que sempre me tratou. Pelas conversas agradáveis e bons momentos compartilhados.

À Profa. Dra. Maria Cristina Borsatto pelo acolhimento, carinho e simpatia que teve para comigo desde comecei a freqüentar a faculdade, mesmo antes de ingressar no mestrado. Pelo exemplo de pessoa justa, humilde e preocupada com o próximo. 
À Profa Dra Raquel Assed Bezerra da Silva pelos momentos agradáveis, pela disposição em ajudar e pela amizade.

À Profa Dra Carla Enoki pelos ensinamentos e incentivos, pelo apoio, pela paciência e pela valiosa colaboração em todos os meus trabalhos.

À Profa Dra Fernanda Weber de Morais Galarreta e Dr. Márcio José Reis pela generosidade, amizade, imprescindivel colaboração na execução deste trabalho.

Aos professores do departamento de Clínica Infantil, Odontologia Preventiva e Social da Faculdade de Odontologia de Ribeirão Preto (USP), Profa. Dra. Sada Assed, Profa. Dra. Léa Assed Bezerra da Silva, Profa. Dra. Aldevina Campos de Freitas, Prof. Dr. Paulo Nelson Filho, Profa. Dra. Alexandra Mussolino de Queiroz, Pof. Dr. Mário Roberto Leonardo, Profa. Dra. Raquel Assed Bezerra da Silva, Profa. Dra. Kranya Diaz Victoria Serrano, Profa. Dra. Maria Cristina Borsatto, Profa. Dra. Mirian Aiko Nakane Matsumoto, Prof. Dr. José Tarcísio Lima Ferreira, Prof. Dr. Adílson Thomazinho, Profa. Dra. Maria Bernadete Sasso Stuani, Profa. Dra. Maria Conceição Pereira Saraiva, Prof. Dr. Fábio Lourenço Romano e Profa. Dra. Andiara de Rossi.

À minhas amigas Fabiana, Camila, Roberta e Vanessa por todas as experiências compartilhadas, pela alegria, carinho e paciência que sempre tiveram comigo, por todos os conselhos, conversas e momentos inesquecíveis, pela amizade de todos esses anos.

À minhas amigas Tais Helena Grecchi e Marcela Cristina Damião Andruciolli por toda a ajuda no desenvolvimento do meu trabalho, pelo carinho, amizade, ótima companfia dentro e fora da faculdade. Vocês são pessoas que admiro e torço muito!

Aos meus amigos e colegas da pós-graduação Camila Scatena, Cíntia Guimarães de Almeida, Danielle Torres Azevedo, Marcela Martín Del Campo Fierro, Marília Pacífico Lucisano, Paula Dariana Fernandes Ferreira, Rodrigo Alexandre Valério, Talitha de Siqueira Mellara pelos momentos compartilhados, agradável convivênvia e amizade.

Aos funcionários do departamento de Clínica Infantil, Odontologia Preventiva e Social da Faculdade de Odontologia de Ribeirão Preto (USP), Michele Cristina Leite Rovanholo, Filomena Leli Placciti, Francisco Wanderley Garcia de Paula e Silva, Carolina Paes Torres Mantovani, Marco Antonio dos Santos, Nilza Leticia Magalhães, Fátima Aparecida Rizoli, Fátima Aparecida Jacinto Daniel, Renata Aparecida Fernandes e Matheus Morelli Zanela, pela paciência, ajuda e cordialidade.

Aos funcionários do Centro do Respirador Bucal do Hospital das Clínicas de Ribeirão Preto, Edna $\mathcal{M}^{a}$ Lege Ferreira, Cleide $\mathcal{M}^{a}$ C. Palucci, Joice G. Tritola, Maria Helena de Andrade, Celia Regina B. Souza por terem me acolhido, pela agradável convivência e carinho. 
Ao Dr Vlísses Menezes, à Leila Azevedo de Almeida e ao Bruno Vieira que também fazem parte da equipe do centro do respirador bucal pela colaboração em todos os trabalhos, pela oportunidade de poder aprender com vocês e pela sempre agradável companfia.

Ao Conselho $\mathcal{N a c i o n a l}$ de Desenvolvimento Científico e Tecnológico, pelo suporte científico e financeiro para realização deste trabalho. 


\section{RESUMO}

CARMO, LA. Área da nasofaringe após a expansão rápida da maxila por meio de radiografia cefalométrica lateral e ressonância magnética. [dissertação] Ribeirão Preto (SP): Faculdade de Odontologia de Ribeirão Preto (FORP) - Universidade de São Paulo; 2011.

A respiração bucal é uma condição frequente na infância, e acredita-se que ao longo do tempo, ela possa influenciar o padrão de desenvolvimento das estruturas craniofaciais, promovendo alterações importantes da oclusão dentária, como deficiência transversal da maxila e mordida cruzada posterior. A expansão rápida da maxila (ERM) é o procedimento ortodôntico/ortopédico mais utilizado para o tratamento dessa maloclusão, principalmente em pacientes em fase de crescimento. O objetivo deste estudo foi verificar o efeito da expansão rápida da maxila sobre a dimensão do espaço nasofaríngeo por meio da radiografia cefalométrica lateral $(R C L)$ e da imagem de ressonância magnética (IRM) e comparar e correlacionar os dados obtidos nos dois exames de imagem. Foram selecionadas trinta crianças, de ambos os gêneros, com idade variando entre 07 e 10 anos, com respiração bucal e/ou mista, em fase de dentição mista, com mordida cruzada posterior uni ou bilateral, envolvendo caninos e molares decíduos e primeiros molares permanentes, que não tivessem sido submetidas a tratamento otorrinolaringológico ou ortodôntico. A respiração bucal foi diagnosticada por meio da nasofibroscopia e os pacientes foram submetidos à ERM com o aparelho disjuntor de Haas modificado. A área da nasofaringe foi avaliada nas radiografias cefalométricas laterais e na ressonância magnética em 2 tempos: antes $\left(T_{1}\right)$ e 6 meses após $\left(T_{2}\right)$ a expansão, utilizando o método de Linder-Aronson e Henrikson (1973). Os resultados mostraram que houve aumento estatisticamente significante da área da nasofaringe após a ERM quando avaliadas as imagens obtidas por meio da ressonância magnética, porém, quando as imagens foram avaliadas por meio da radiografia cefalométrica lateral, não houve aumento estatisticamente significante. Houve correlação positiva entre a área da nasofaringe medida por meio da IRM e RCL, pré e pósexpansão. Pôde-se concluir que não houve alteração significativa na dimensão do espaço nasofaríngeo após a ERM, avaliado por meio da RCL, porém, com a IRM o aumento desse espaço foi significativo. Além disso, houve correlação positiva entre os dados obtidos nos dois exames de imagem, porém, quando comparados apresentaram diferença estatisticamente significante.

Palavras-chave: Imagem por Ressonância Magnética; Radiografia Cefalométrica Lateral; Nasofaringe; Técnica de Expansão Palatina. 


\begin{abstract}
CARMO, LA. Nasopharyngeal area after the rapid maxillary expansion by lateral cephalometric radiography and magnetic resonance imaging. [dissertation]. Ribeirão Preto (SP): Faculdade de Odontologia de Ribeirão Preto (FORP) - Universidade de São Paulo; 2011.
\end{abstract}

Mouth breathing is an extremely common condition in childhood, and it is believed that over time, it can influence the pattern of development of craniofacial structures, leading to major changes in dental occlusion, as transverse maxillary deficiency and posterior crossbite. The rapid maxillary expansion (RME) is the orthodontic / orthopedic procedure most used to treat this malocclusion, especially in growing patients. The aim of this study was to evaluate the effects of rapid maxillary expansion in nasopharyngeal dimensions by lateral cephalometric radiography and magnetic resonance imaging and to compare and to correlate the results of the two images exams. Thirty children of both genders were selected, ranging in age from 07-10 years, with buccal and/or mixed breathing, in mixed dentition phase, using unilateral or bilateral posterior crossbite, involving canine and molars deciduous and first permanent molars, without any type of previous orthodontic or otolaryngology treatment. The diagnosis of oral breathing was made by nasofibroscopy and the patients were submitted to RME by modified expander of Haas. The nasopharynx area was evaluated by lateral cephalometric radiography (LCR) and magnetic resonance imaging (MRI) in two times: before the expansion and 6 months after it, according to the method described by Linder-Aronson and Henrikson (1973). The results showed a statistically significant increase of the nasopharyngeal area after the expansion when evaluated images were obtained by magnetic resonance imaging, but a not statistically significant increase was observed when the images were evaluated by lateral cephalometric radiography. A positive correlation was found between the nasopharynx area measure by MRI and LCR, before and after expansion. It was concluded that no significant changes in the nasopharyngeal dimensions were found after the RME evaluated by LCR, however, the increase of this area was significant by the MRI. Furthermore, there was a positive correlation between the values of the two images exams, however, when they were compared a statistically significant difference was demosntrated.

Keywords: Magnetic Resonance Imaging; Lateral Cephalometric Radiograph; Nasopharynx; Palatal Expansion Technique. 


\section{LISTA DE ILUSTRAÇÕES}

Figura 1 - Nasofibroscopia (A: aparelho e B: exame)

Figura 2 - Vista oclusal do arco dentário superior (A) e vista oclusal do disjuntor de Haas modificado (B).

Figura 3 - Vista frontal antes da correção da mordida cruzada posterior (A) e vista frontal após a correção (B)

Figura 4 - Vista oclusal do disjuntor de Haas modificado logo após a sua instalação (A) e 6 meses após a expansão (B)....

Figura 5 - Radiografia oclusal da maxila antes da correção da mordida cruzada posterior (A) e radiografia oclusal logo após as ativações (B)

Figura 6 - Radiografia oclusal 6 meses após a obtenção da expansão rápida da maxila......

Figura 7 - Radiografia cefalométrica lateral...

Figura 8 - Traçado cefalométrico em norma lateral, pontos cefalométricos e área da nasofaringe....30

Figura 9 - Ressonância magnética:equipamento.

Figura 10 - Imagem de ressonância magnética.

Figura 11 - Área da nasofaringe em imagem de ressonância magnética.

Figura 12 - Média da área da nasofaringe das crianças submetidas à radiografia cefalométrica lateral, antes e após a ERM...

Figura 13 - Média da área da nasofaringe das crianças submetidas à ressonância magnética, antes e após a ERM.

Figura 14 - Correlação entre a área da nasofaringe por meio da IRM e radiografia cefalométrica lateral antes da ERM.

Figura 15 - Correlação entre a área da nasofaringe por meio da IRM e radiografia cefalométrica lateral após a ERM...

Figura 16 - Comparação entre as medidas da área da nasofaringe por meio da radiografia cefalométrica lateral e da ressonância magnética antes da ERM.

Figura 17 - Comparação entre as medidas da área da nasofaringe por meio da radiografia cefalométrica lateral e da ressonância magnética 180 dias após a ERM. 


\section{LISTA DE TABELAS}

Tabela 1 - Médias, desvios padrão (DP), valores mínimo (Mín) e máximo (Máx) das medidas da área da nasofaringe obtidas pela ressonância magnética e pela radiografia cefalométrica lateral, realizadas nos momentos $\mathrm{T}_{1}$ e $\mathrm{T}_{2}$

Tabela 2 - Comparação das medidas da área da nasofaringe obtidas pela ressonância magnética e pela radiografia cefalométrica lateral, realizadas nos momentos $T_{1}$ e $T_{2}$ 


\section{SUMÁRIO}

1. INTRODUÇÃO

2. PROPOSIÇÃO

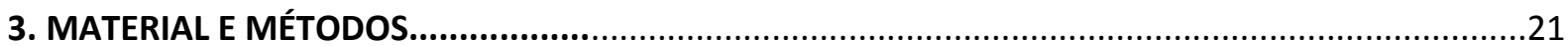

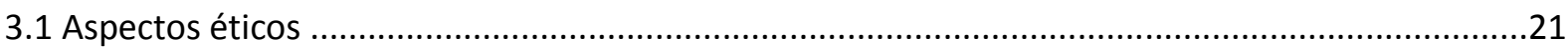

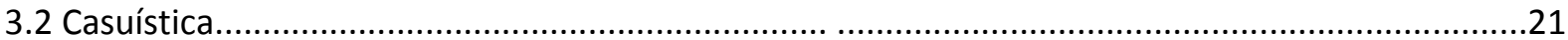

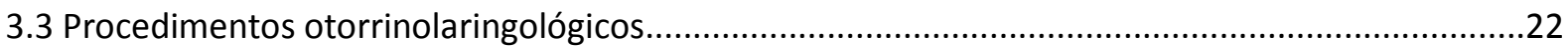

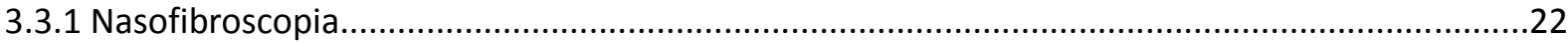

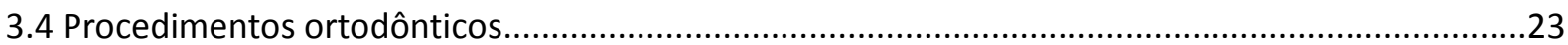

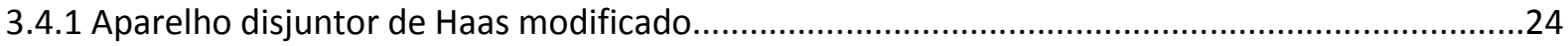

3.4.1.1 Material, confecção, instalação e ativação do disjuntor de Haas.............................................24

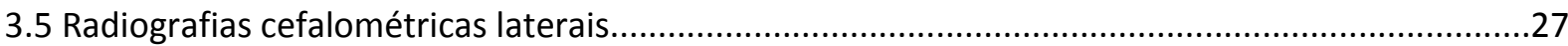

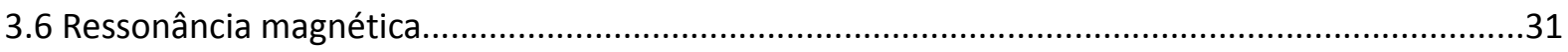

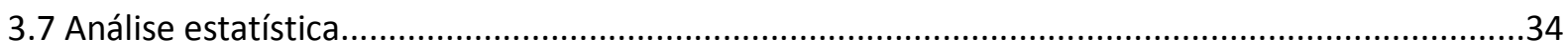

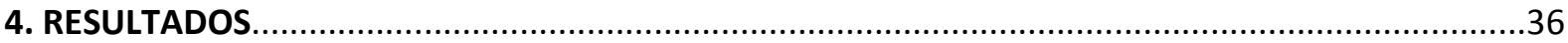

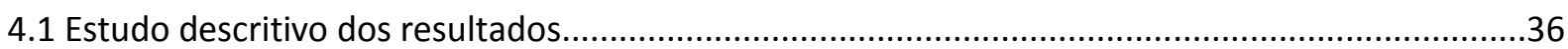

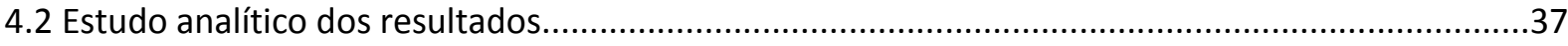

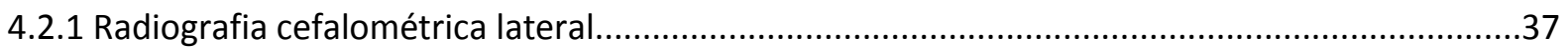

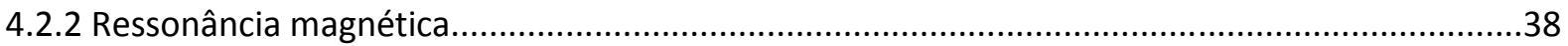

4.2.3 Correlação e comparação entre área da nasofaringe pré e pós-ERM por meio dos exames de ressonância magnética e radiografia cefalométrica lateral................................................................38

5. DISCUSSÃO

6. CONCLUSÃO

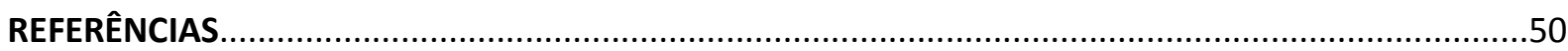

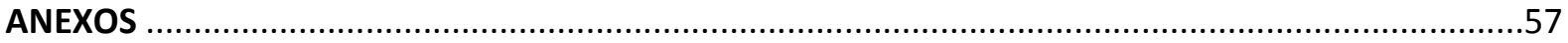


INTRODUÇÃO 


\section{INTRODUÇÃO}

A influência do padrão respiratório no crescimento e desenvolvimento craniofacial tem sido amplamente abordada e discutida na literatura, uma vez que a respiração bucal seria uma adaptação do sistema estomatognático a algum fator obstrutivo ou funcional que leva a inúmeras alterações estruturais e posturais (Krakauer e Guilherme, 2000; Lessa et al., 2005; Branco et al., 2007). Considera-se como respirador bucal aquele que possui um desvio do padrão de respiração normal, sendo esta, por vezes, insuficiente, passando a ser substituída pela suplência bucal ou mista (Lusvarghi, 1999).

A respiração bucal possui etiologia multifatorial, tendo como uma das causas mais comuns a obstrução nasal, que é caracterizada pela obstrução mecânica da passagem aérea com consequente aumento da resistência nasal. Em crianças, esse quadro tem como principais causas a rinite e a hiperplasia da tonsila faríngea e/ou palatina como fatores mecânicos responsáveis por essa restrição à passagem aérea (Souza et al., 2000; Carvalho, 2003; Bahadir, 2006).

As tonsilas geralmente sofrem hiperplasia entre 3 e 6 anos de idade (Linder-Aronson e Leighton, 1983; Vilella et al., 2006; Ysunza et al., 2008), regredindo, de maneira progressiva até os 10-11 anos quando há discreto aumento e, então, tornam a regredir continuamente (Vilella et al., 2006).

A relação da função respiratória com o desenvolvimento da oclusão e da morfologia craniofacial apresenta resultados controversos na literatura (McNamara, 1981; Waren e Spalding, 1991; Yamada et al., 1997; Vig, 1998) e uma vez que o padrão de crescimento dentoalveolar é complexo e afetado por fatores genéticos, ambientais e comportamentais, o debate a respeito do crescimento e do modo respiratório deve continuar até que todas essas variáveis e suas correlações sejam determinadas (Crouse et al., 2000).

Segundo a Teoria da Matriz Funcional de Moss (1969), o crescimento ósseo é influenciado quase que exclusivamente pela função, portanto, o crescimento facial está intimamente associado à atividade funcional dos diferentes componentes da cabeça e pescoço. A respiração nasal associada a outras funções como mastigação e deglutição propiciam adequado desenvolvimento do complexo craniofacial. Alterações prolongadas no padrão respiratório levarão a inúmeras repercussões no sistema estomatognático, 
conduzindo a alteração desfavorável no complexo craniofacial, com o desenvolvimento de um conjunto de modificações funcionais, dentoalveolares e esqueléticas (Valera et al., 2003; Thomazinho et al., 2005). No período ativo de crescimento, o impacto negativo desse padrão respiratório no desenvolvimento de alterações esqueléticas e de posicionamento dentário é acentuado (Principato, 1991).

A ocorrência de deformidades maxilares associadas a problemas respiratórios têm sido o foco de muitos estudos, sendo a deficiência maxilar transversa a mais frequente. $\mathrm{O}$ arco dentário superior é vulnerável às mudanças provenientes da respiração bucal, alterando-se morfologicamente, uma vez que, perde sua conformação parabólica normal para assumir uma forma triangular, com a base voltada para região posterior, caracterizando a atresia do arco dentário superior e culminando com o quadro clínico reconhecido como mordida cruzada posterior (Capelozza Filho e Silva Filho, 1997; Faria et al., 2002; Mattar et al., 2004; Enoki et al., 2006; Galarreta, 2010).

A expansão maxilar, também chamada expansão rápida da maxila (ERM) é o procedimento indicado para pacientes com essa deficiência, sendo muito usado em Ortodontia, cujo objetivo principal é promover a separação dos ossos maxilares (disjunção maxilar) com abertura da sutura palatina mediana e consequentemente aumentando a dimensão transversal da maxila (Haas, 1961). Seu efeito não é limitado ao arco dentário superior, uma vez que, a maxila está conectada a dez ossos do complexo craniofacial e, portanto, a ERM pode afetar direta ou indiretamente estruturas como cavidade nasal, faringe, articulação temporomandibular, ouvido médio e processo pterigóide do osso esfenóide (Cleylan et al., 1996; Enoki et al., 2006; Lione et al., 2008; Galarreta, 2010; Matsumoto et al., 2010; Langer et al., 2011).

A força aplicada aos dentes e processo alveolar excede o limite necessário para a movimentação ortodôntica e promove separação dos ossos maxilares, sendo por isso considerado um procedimento ortopédico. Após o pico de crescimento puberal, a maturação óssea é completa e a sutura palatina mediana é ossificada, causando resistência esquelética maior ao procedimento ortopédico, sendo necessária sua associação com procedimentos cirúrgicos para obtenção de resultados satisfatórios (Kiliç e Oktay, 2008).

A maior resistência à expansão não está na sutura palatina mediana, mas sim nas estruturas adjacentes à maxila, particularmente nos ossos zigomático e esfenóide, que 
promovem o afastamento dos maxilares lateralmente em direção jugal (Bishara e Stanley, 1987).

As mudanças dentoalveolares e craniofaciais produzidas pela ERM ocorrem tanto no plano transversal como nos planos vertical, frontal e sagital, sendo altamente favoráveis ao desenvolvimento craniofacial (Haas, 1961; Capelozza Filho e Silva Filho, 1997; Moore, 1997; Chung e Font, 2004).

Tem sido relatado na literatura que uma das vantagens adicionais da ERM seria a melhora na respiração, uma vez que, com esse procedimento as conchas nasais inferiores são deslocadas lateralmente com recolocação das paredes internas da cavidade nasal, promovendo o abaixamento da abóboda palatina, corrigindo o septo nasal e consequentemente melhorando a respiração (Goldsmith e Stool, 1994; Capelozza Filho e Silva Filho, 1997; Enoki et al., 2006; Matsumoto et al., 2010; Langer et al., 2011).

Muitos estudos foram realizados a fim de avaliar os efeitos da ERM na geometria da cavidade nasal assim como na resistência aérea e fluxo nasal, mas poucos enfocam seu efeito nas dimensões do espaço nasofaríngeo. Nesse sentido, diferentes modalidades de imagens têm sido usadas para avaliar a via aérea superior e as estruturas ósseas e de tecido mole adjacentes, incluindo a radiografia cefalométrica, a tomografia computadorizada e a imagem por ressonância magnética (Schwab e Goldeberg, 1998; Pereira et al., 2001; SantosPinto et al., 2006).

Linder-Aronson (1970) foi o primeiro a analisar cefalometricamente o espaço nasofaríngeo em crianças com respiração bucal e nasal e desde então, outros autores têm feito essa análise por meio de diferentes metodologias.

Em se tratando de métodos de diagnóstico é importante ressaltar a radiografia cefalométrica lateral, um exame radiográfico padrão da região de cabeça e pescoço, que é geralmente usada para propósitos de diagnóstico em Ortodontia e para analisar os resultados dos procedimentos ortodônticos. Tem também sido indicada para avaliação dos seguintes fatores: mudanças dentais e esqueléticas induzidas pela ERM (Chung e Font, 2004); modificações induzidas pela ERM no espaço nasal posterior e anterior (da Silva Filho et al., 1995; Basciftci et al., 2002; Chung e Font, 2004; Enoki et al., 2006); e modificações dos espaços da oro e nasofaringe (Baik et al., 2002; Kiliç e Oktay, 2008; Monini et al., 2009).

As radiografias cefalométricas apresentam suas limitações no que diz respeito às mensurações, uma vez que, estas são projeções de estruturas tridimensionais que compõem 
a face e o crânio em um plano bidimensional, com informações limitadas sobre o espaço nasofaríngeo. $O$ fato da radiação $X$ propagar-se em linha reta e sob forma de um feixe divergente contribui para produzir distorções na imagem final, o que poderia comprometer a confiabilidade do diagnóstico obtido por meio da cefalometria (Schwab e Goldeberg, 1998).

Em contrapartida, pesquisas já consagraram valores normais para os padrões esqueléticos, dentários e dimensões das vias aéreas superiores, além do que a radiografia cefalométrica apresenta vantagens de ser a técnica de radiografia plana que apresenta os menores índices de distorção, pode ser reprodutível com ajuda de cefalostatos e ainda ser de fácil acesso e baixo custo. Os ortodontistas em geral não utilizam rotineiramente exames como tomografia computadorizada ou ressonância magnética, pelo alto custo financeiro e de tempo e pela alta exposição à radiação no caso da tomografia (Lowe, 1993; Bascifciti et al., 2002).

Em se tratando de vias aéreas superiores, alguns autores referem que a imagem de ressonância magnética (IRM) é um método preciso para descrever as variações no tamanho das tonsilas palatinas e faríngeas, uma vez que, permite a ampliação de imagens, não há sobreposição de estruturas, além da vantagem de ser livre de radiação ionizante (Jaw et al., 1999; Vogler et al., 2000; Donnelly et al., 2002a). Para a avaliação dos tecidos moles, este método parece ser superior à radiografia cefalométrica lateral, pois, estruturas tridimensionais podem ser retratadas com limitações nas radiografias cefalométricas. Protocolos padronizados foram desenvolvidos para a realização de medições das vias aéreas superiores por meio da ressonância magnética. Em comparação com a tomografia computadorizada, a ressonância magnética não expõe o paciente à radiação ionizante, portanto, o número de exames realizados em um sujeito é, teoricamente, ilimitado (Stuck et al., 2002).

Assim, como os trabalhos encontrados na literatura para avaliar as vias aéreas superiores após disjunção maxilar têm utilizado as radiografias cefalométricas laterais e os mais recentes, técnicas de imagem como a ressonância magnética, decidimos estudar o efeito da ERM sobre a região nasofaríngea por meio dos dois exames de imagens e efetuar a comparação e a correlação dos resultados encontrados. 
PROPOSIÇÃO 


\section{PROPOSIÇÃO}

Os objetivos deste trabalho foram:

2.1 - Verificar o efeito da expansão rápida da maxila sobre a dimensão do espaço nasofaríngeo por meio da radiografia cefalométrica lateral e da ressonância magnética;

2.2 - Comparar e correlacionar os dados obtidos nos dois exames de imagem. 
MATERIAL E MÉTODOS 


\section{MATERIAL E MÉTODOS}

\subsection{ASPECTOS ÉTICOS}

O presente projeto foi submetido à avaliação pelo Comitê de Ética em Pesquisa do Hospital das Clínicas da Faculdade de Medicina de Ribeirão Preto da Universidade de São Paulo (HC/FMRP-USP) de acordo com o processo no 13658/2006 (Anexo A).

Os responsáveis pelas crianças que apresentaram os requisitos necessários para constituir a amostra do presente estudo foram convidados a autorizá-las a participar da pesquisa, sendo esta participação absolutamente voluntária. Foi assegurado ao responsável no momento do convite, que caso ele não concordasse com a participação da criança, tal decisão não traria nenhum prejuízo para ambos, bem como haveria total liberdade de retirar seu consentimento a qualquer momento no transcorrer da pesquisa e de deixar de participar do estudo.

O termo de consentimento livre e esclarecido foi lido para todos os responsáveis pelas crianças (Anexo B), fornecendo-lhes informações sobre o objetivo, riscos e benefícios do estudo do qual as crianças participariam. Foram aceitos no estudo somente voluntários cujos pais e/ou responsáveis assinaram o termo de consentimento informado, previamente aprovado pelo Comitê de Ética de acordo com a resolução 196/96 do Conselho Nacional de Saúde.

Foi garantida a segurança de não serem identificados, bem como de que todas as informações fornecidas por eles seriam mantidas sob caráter confidencial.

\subsection{CASUÍSTICA}

Para a realização do estudo a amostra consistiu de 30 pacientes, de ambos os gêneros, com idade variando entre 07 e 10 anos, em fase de dentição mista, com mordida cruzada posterior, uni ou bilateral, envolvendo caninos e molares decíduos e primeiros molares permanentes. Estes pacientes foram selecionados na Triagem da Clínica de Ortodontia Preventiva e Interceptora da Faculdade de Odontologia de Ribeirão Preto - Universidade de São Paulo (USP) e no Centro do Respirador Bucal (CERB) da Divisão de Otorrinolaringologia 
do Hospital das Clínicas da Faculdade de Medicina de Ribeirão Preto - Universidade de São Paulo (HC/FMRP-USP).

Os pacientes deveriam apresentar respiração bucal e /ou mista, com diagnóstico otorrinolaringológico realizado previamente no Centro do Respirador Bucal da Faculdade de Medicina de Ribeirão Preto - Universidade de São Paulo. Foram excluídos pacientes que apresentassem distúrbios sistêmicos, estivessem sob medicação antibiótica, utilizassem sistemicamente medicação nasal de uso tópico ou sistêmico, que haviam feito cirurgia de palato, ou já tivessem sido submetidos a tratamento ortodôntico prévio.

\subsection{PROCEDIMENTOS OTORRINOLARINGOLÓGICOS}

Após avaliação completa dos pacientes por um otorrinolaringologista a fim de obter o diagnóstico clínico de respiração bucal e/ou mista, foi realizado exame de nasofibroscopia (rinoscopia anterior e posterior) com o objetivo de avaliar o trofismo (normotróficas, hipertróficas ou hipotróficas) e a coloração (pálidas, coradas ou hiperemiadas) das conchas nasais inferiores, o grau de obstrução das coanas pelo tecido adenoideano e a presença de deformidades septais. A nasofibroscopia foi realizada apenas antes da expansão rápida da maxila $\left(T_{1}\right)$, uma vez que, seu objetivo foi auxiliar no diagnóstico e seleção dos pacientes.

\subsubsection{NASOFIBROSCOPIA}

Essa técnica consiste da inspeção visual das fossas nasais com mais riqueza de detalhes, com ótimas condições de luminosidade e nitidez de imagens. Foram observadas as características macroscópicas quando uma microcâmera e uma fonte de luz se acoplaram à extremidade da fibra óptica flexível ou rígida (de $4 \mathrm{~mm} 0^{\circ}$ ) da marca STORZ, de aproximadamente $15 \mathrm{~cm}$ de comprimento (Figura 1A). Foi possível a avaliação precisa das estruturas anatômicas na sua forma e cor, assim como a presença de alterações patológicas (hipertrofia das conchas nasais inferiores, médias e superiores, hiperplasia adenoideana, massas nasais e desvios septais).

O exame é feito com o paciente sentado e sob anestesia local, feita pelo otorrinolaringologista, com a inserção de uma mecha de algodão, em cada uma das narinas do paciente, embebido de anestésico (neototucaína, associado com efedrina a $0.25 \%$ ), por 
aproximadamente 10 minutos. Após a remoção do algodão, o endoscópio foi gradativamente inserido na narina, e por intermédio da imagem refletida na televisão e gravada em vídeo, foi inspecionada e diagnosticada a presença de anormalidades, quando presentes, nas fossas nasais e/ou nasofaringe (Figura 1B). O endoscópio é introduzido na cavidade nasal do paciente seguindo três áreas de observação, a primeira ao longo do assoalho nasal; a segunda entre o septo e as conchas nasais inferior e média, até a coana, quando a nasofaringe é avaliada; e a terceira quando o endoscópio é retirado entre a concha média e a parede lateral do nariz, ou seja, no meato médio. Este procedimento foi realizado para ambas as narinas.

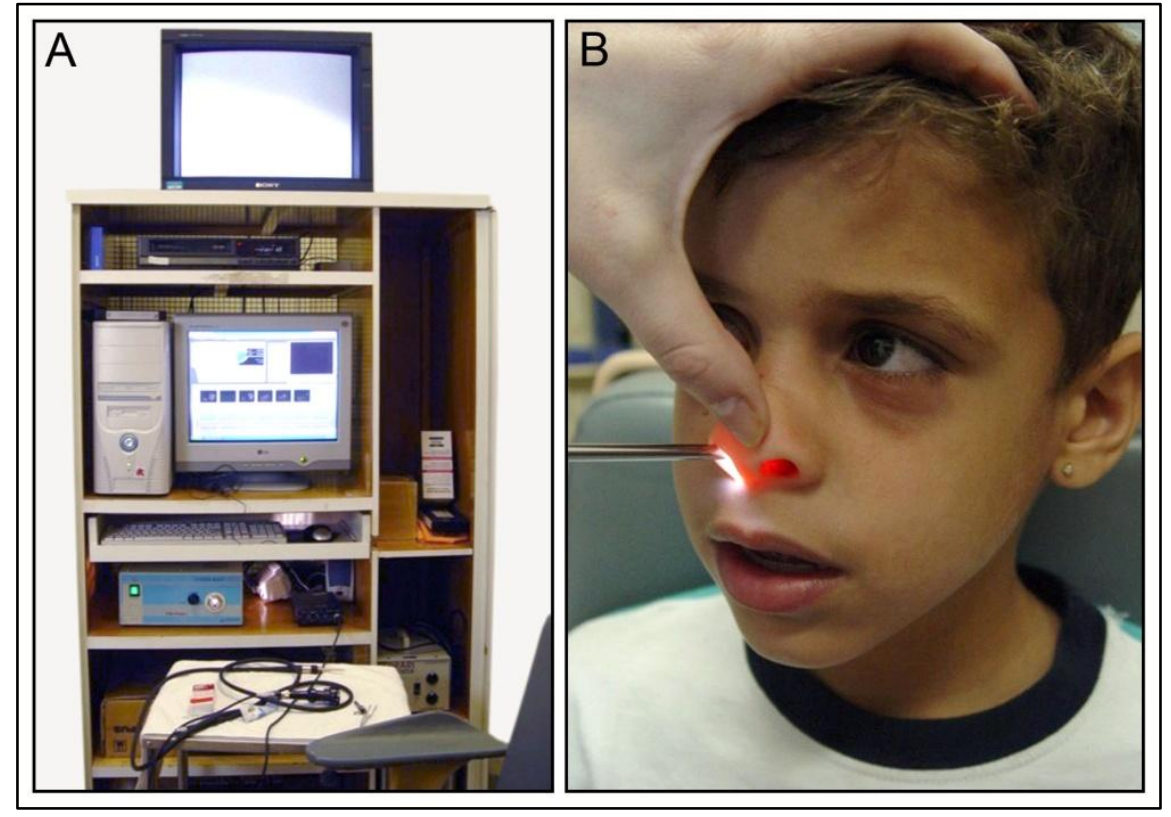

Figura 1 - Nasofibroscopia (A: aparelho e B: exame).

\subsection{PROCEDIMENTOS ORTODÔNTICOS}

A documentação ortodôntica completa a qual inclui radiografias cefalométricas laterais e oclusais, modelos de estudo, fotografias intrabucais e fotografias extrabucais de frente e de perfil foi realizada antes $\left(T_{1}\right)$ e 6 meses $\left(T_{2}\right)$ após a expansão rápida da maxila. Após análise da documentação inicial e realização do plano de tratamento ortodôntico dos pacientes, procedeu-se à instalação do aparelho ortodôntico dento-muco-suportado Disjuntor de Haas modificado (Haas, 1965) para correção da mordida cruzada posterior uni ou bilateral. A instalação e ativação do aparelho foram efetuadas segundo os princípios descritos por Haas (1961). 


\subsubsection{APARELHO DISJUNTOR DE HAAS MODIFICADO}

O aparelho constituiu de anéis ortodônticos pré-fabricados (GAC 16015 e 26015) adaptados aos primeiros molares permanentes superiores e fios de aço inoxidável de 1,0 $\mathrm{mm}$ de diâmetro (Dentaurum 513-100) contornados às faces vestibulares e palatinas dos primeiros molares permanentes, molares e caninos decíduos, soldados aos anéis ortodônticos com solda de prata. Uma placa de acrílico adaptada ao palato une as estruturas metálicas do lado direito e esquerdo e mantém um parafuso de expansão (Dentaurum 600.303 ) centralizado ao nível da sutura palatina mediana (Figura 2B).

\subsubsection{Material, Confecção, Instalação e Ativação do Disjuntor de Haas}

O material necessário para a confecção do Disjuntor de Haas modificado consistiu de: anéis pré-fabricados (GAC 16015 e 26015), fio de aço inoxidável 1,0 mm (Dentaurum 513.100), parafuso expansor de 7,0 $\mathrm{mm}$ (Dentaurum 600.303), solda de prata em fio 0,38 mm (Unitek 700.102), fluxo para solda de prata (Unitek 700.202) e acrílico incolor autopolimerizável (Ortho Class Clássico).

Separadores elastoméricos da GAC foram utilizados nos molares permanentes por uma semana com a finalidade de promover o afastamento dos mesmos em relação aos segundos molares decíduos. Após esse período, anéis ortodônticos pré-fabricados (GAC 16015 e 26015) foram selecionados. Uma vez corretamente adaptados e em posição procedeu-se à moldagem do arco superior, com alginato Jeltrate Dentisply - Caulk, e então, os anéis foram transferidos ao molde obtido para posterior vazamento com gesso pedra branco Mossoró tipo alfa especial. A partir do modelo de gesso obtido foi confeccionado o aparelho disjuntor de Haas modificado. Com fio de aço inoxidável $1.0 \mathrm{~mm}$ foram confeccionadas duas barras adaptadas às faces vestibulares e palatinas dos primeiros molares permanentes, molares e caninos decíduos, as quais foram soldadas aos anéis dos primeiros molares permanentes com solda de prata. Com o parafuso expansor fixado e centralizado sobre a sutura palatina mediana ao nível do segundo molar decíduo, procedeu-se a acrilização do suporte mucoso do aparelho com acrílico Orto-Class. O limite anterior do aparelho deve liberar as rugosidades palatinas; lateralmente, o aparelho deve se estender até $5.0 \mathrm{~mm}$ da margem da gengiva e, na região posterior, deve incluir os primeiros molares permanentes. Após o 
acabamento e polimento do aparelho, o mesmo foi instalado por meio de cimentação dos anéis com ionômero de vidro - Ketac Cem Easymix (3M) e as barras coladas aos caninos decíduos, com resina composta fotopolimerizável (Transbond XT, 3M).

Após a instalação do aparelho o parafuso expansor foi ativado em uma volta completa $(1,0 \mathrm{~mm})$ seguindo as recomendações do autor (Haas, 1961). Cada ativação (1/4 de volta ou $0,25 \mathrm{~mm}$ ) foi realizada a cada 15 minutos até completar $1,0 \mathrm{~mm}$ de expansão e o responsável pelo paciente foi orientado a ativar diariamente o aparelho com $1 / 4$ de volta a cada 12 horas, até atingir a quantidade total de expansão necessária. O monitoramento do paciente foi realizado a cada 14 dias.

A correção da mordida cruzada posterior uni ou bilateral (Figura 3) foi verificada pelo contato dos planos inclinados das vertentes vestibulares das cúspides palatinas dos primeiros molares superiores com os planos inclinados linguais das cúspides vestibulares dos primeiros molares inferiores, e também pelo aparecimento de um diastema entre os incisivos centrais superiores que confirma, clinicamente, a abertura da sutura palatina mediana.

Após a correção da mordida cruzada, o parafuso expansor foi travado utilizando-se para isso fios de amarrilho $(0,12 \mathrm{~mm})$ e o aparelho foi então mantido como contenção, sem ativação, por um período de 180 dias para permitir a reorganização das fibras e a remodelação óssea da sutura palatina mediana após a expansão, como já observado por outros autores (Bishara e Stanley, 1987; da Silva Filho et al., 2006; Lione et al., 2008; Ballanti et al., 2010; Franchi et al., 2010).

Na figura 4 observa-se o disjuntor de Haas modificado logo após sua instalação e 6 meses após a expansão.

As radiografias oclusais foram obtidas antes da instalação do expansor (Figura 5A), logo após concluídas as ativações e travamento do parafuso expansor (Figura 5B) e 6 meses após remoção (Figura 6).

Uma vez terminado o período de contenção, o aparelho disjuntor foi removido e um aparelho de contenção removível superior com grampos vestibulares de retenção e placa palatina de acrílico foi instalado para estabilização da oclusão e da musculatura perioral. 


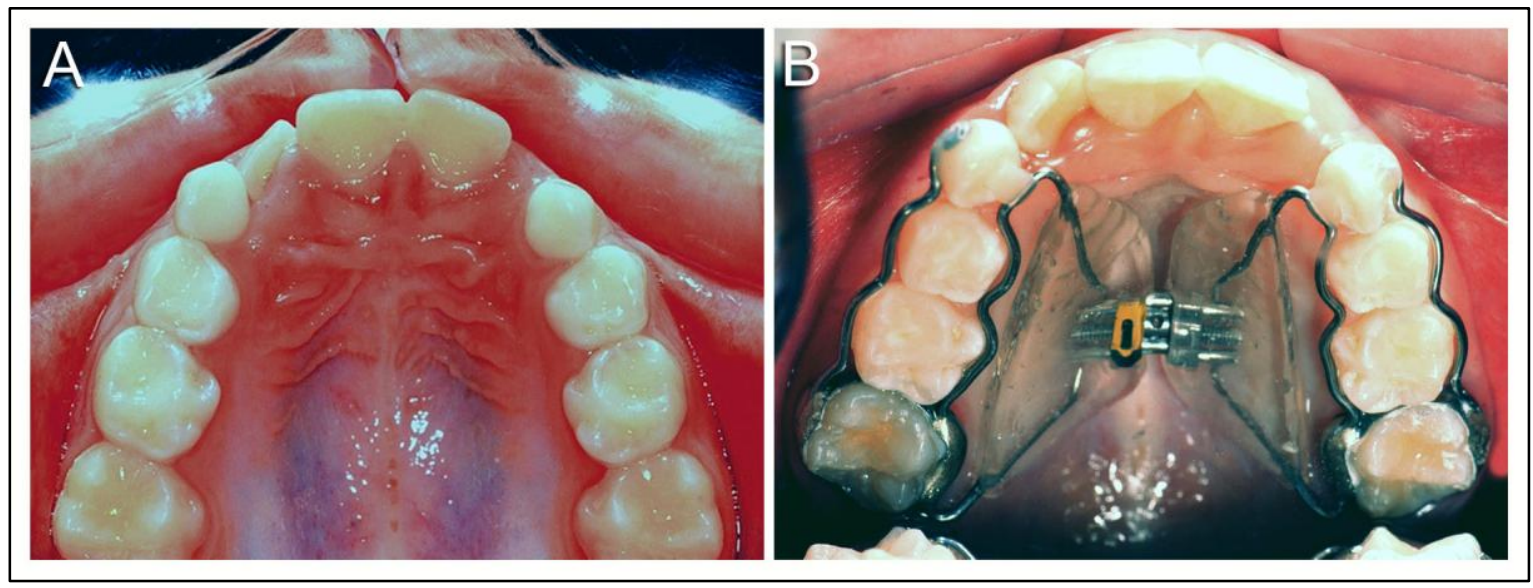

Figura 2 - Vista oclusal do arco dentário superior $(A)$ e vista oclusal do disjuntor de Haas modificado (B).

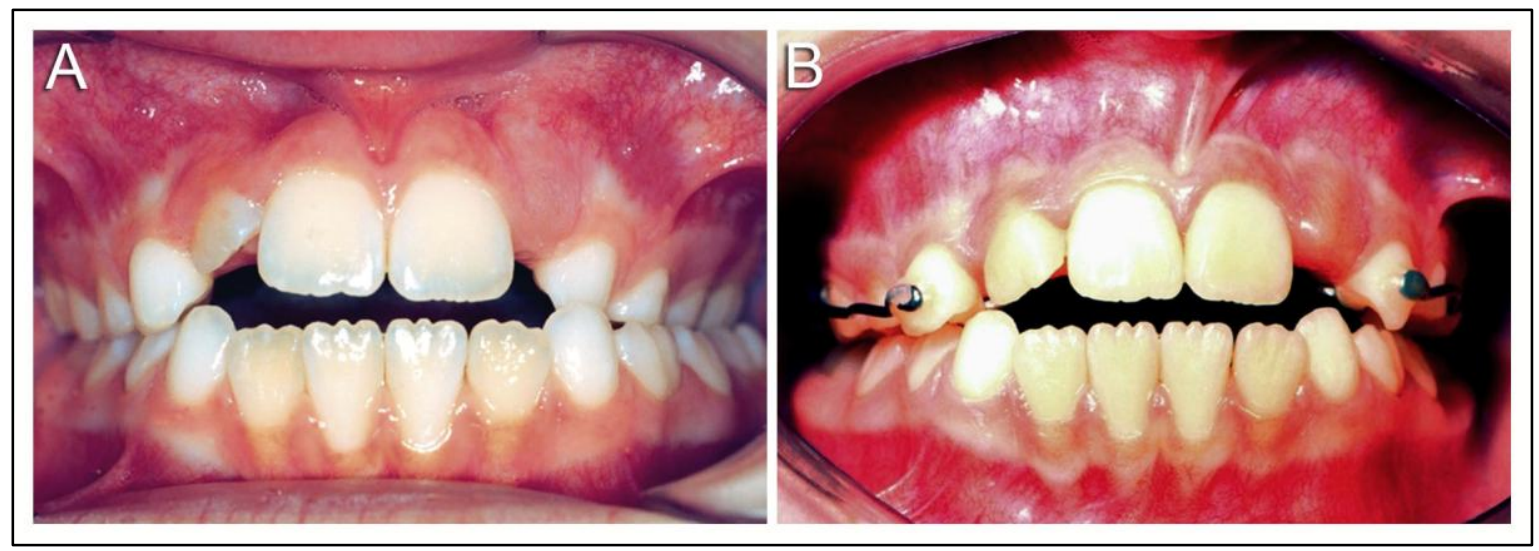

Figura 3 - Vista frontal antes da correção da mordida cruzada posterior $(A)$ e vista frontal após a correção (B).

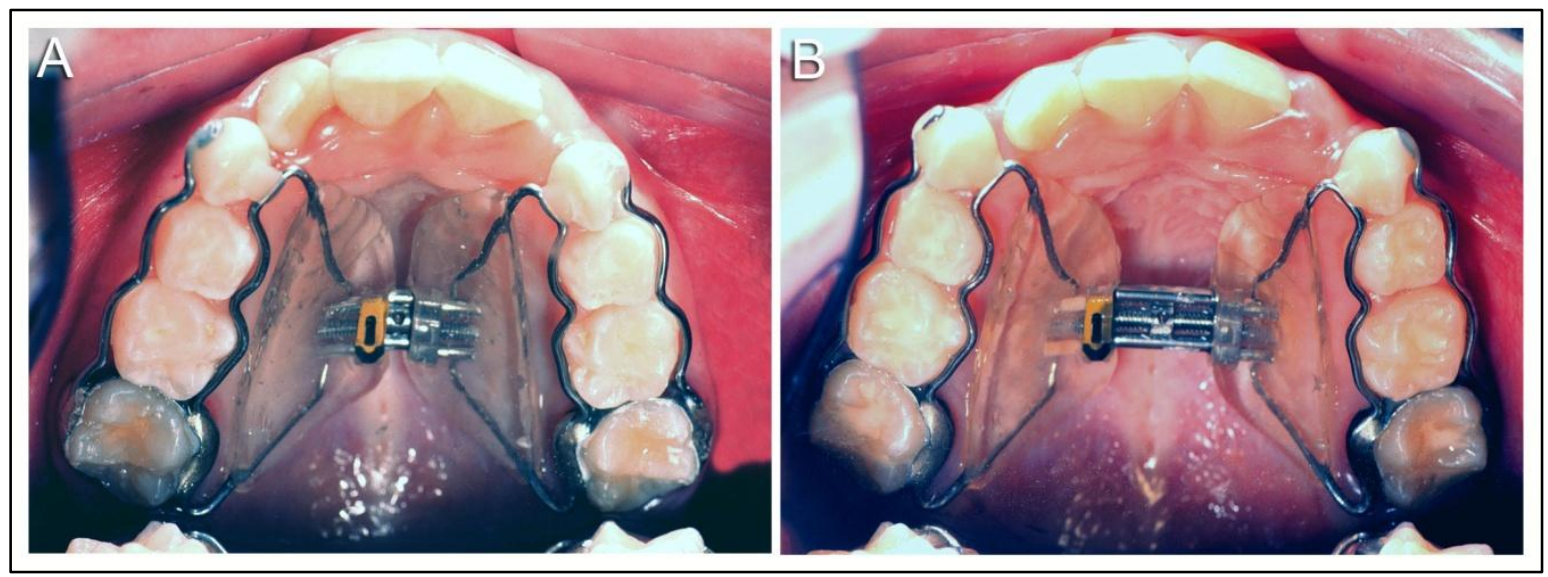

Figura 4 - Vista oclusal do disjuntor de Haas modificado logo após a sua instalação (A) e 6 meses após a expansão (B). 


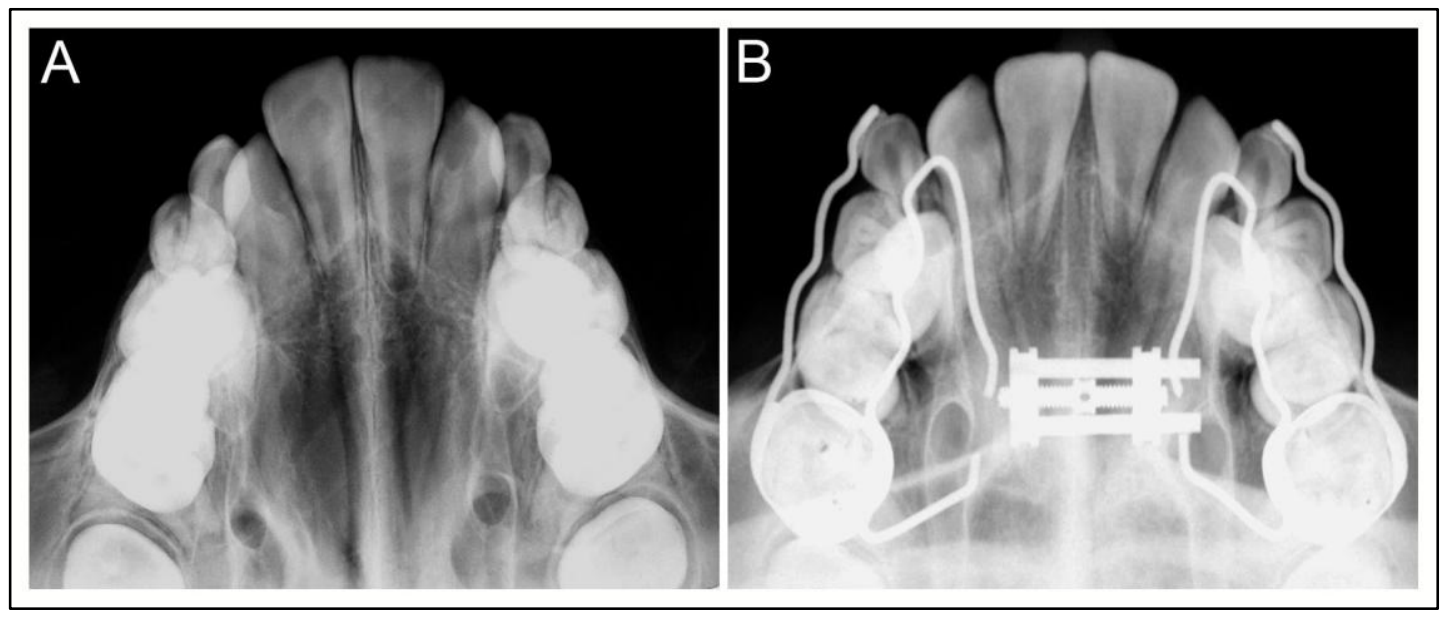

Figura 5 - Radiografia oclusal da maxila antes da correção da mordida cruzada posterior (A) e radiografia oclusal logo após as ativações (B).

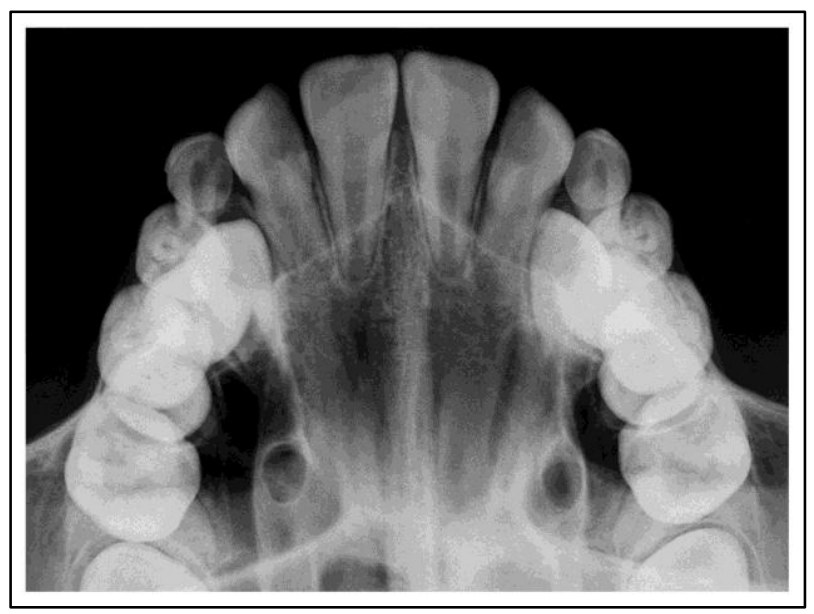

Figura 6 - Radiografia oclusal 6 meses após a obtenção da expansão rápida da maxila.

\subsection{RADIOGRAFIAS CEFALOMÉTRICAS LATERAIS}

As radiografias cefalométricas laterais foram realizadas em equipamento Siemens Orthophos, com a cabeça da criança imobilizada no cefalostato e orientada com o plano horizontal de Frankfurt.

As imagens obtidas (Figura 7) foram digitalizadas via scanner em arquivo digital, transferidas para um computador para localização dos pontos e posterior leitura das dimensões da área da nasofaringe pelo software Cad Overlay (versão 2000). Todas as radiografias foram digitalizadas e analisadas pelo mesmo pesquisador.

As radiografias cefalométricas laterais permitiram avaliar a área da nasofaringe nos dois tempos propostos no estudo: antes $\left(T_{1}\right)$ e 6 meses após $\left(T_{2}\right)$ a expansão rápida da maxila. Os parâmetros utilizados por essa técnica para avaliar a área da nasofaringe 
basearam-se nos pontos anatômicos e medidas cefalométricas descritos segundo LinderAronson e Henrikson (1973), conforme mostra a figura 8.

- Os pontos anatômicos:

S (Sela) - ponto situado no centro geométrico da sela turca do osso esfenóide;

Ba (Básio) - ponto mais posterior e inferior do osso occipital na margem anterior do forame magno;

ENP (Espinha nasal posterior) - vértice da espinha nasal posterior;

So - ponto médio da distância Sela-Básio;

$\mathrm{Ad}_{1}$ - interseccção da linha ENP-So pela parede posterior da nasofaringe;

$\mathrm{Ad}_{2}$ - intersecção da linha ENP-Básio pela parede posterior da nasofaringe.

Medida cefalométrica (Figura 9):

$\mathrm{Ad}_{1}-\mathrm{Ad}_{2}$-ENP: área da nasofaringe. 


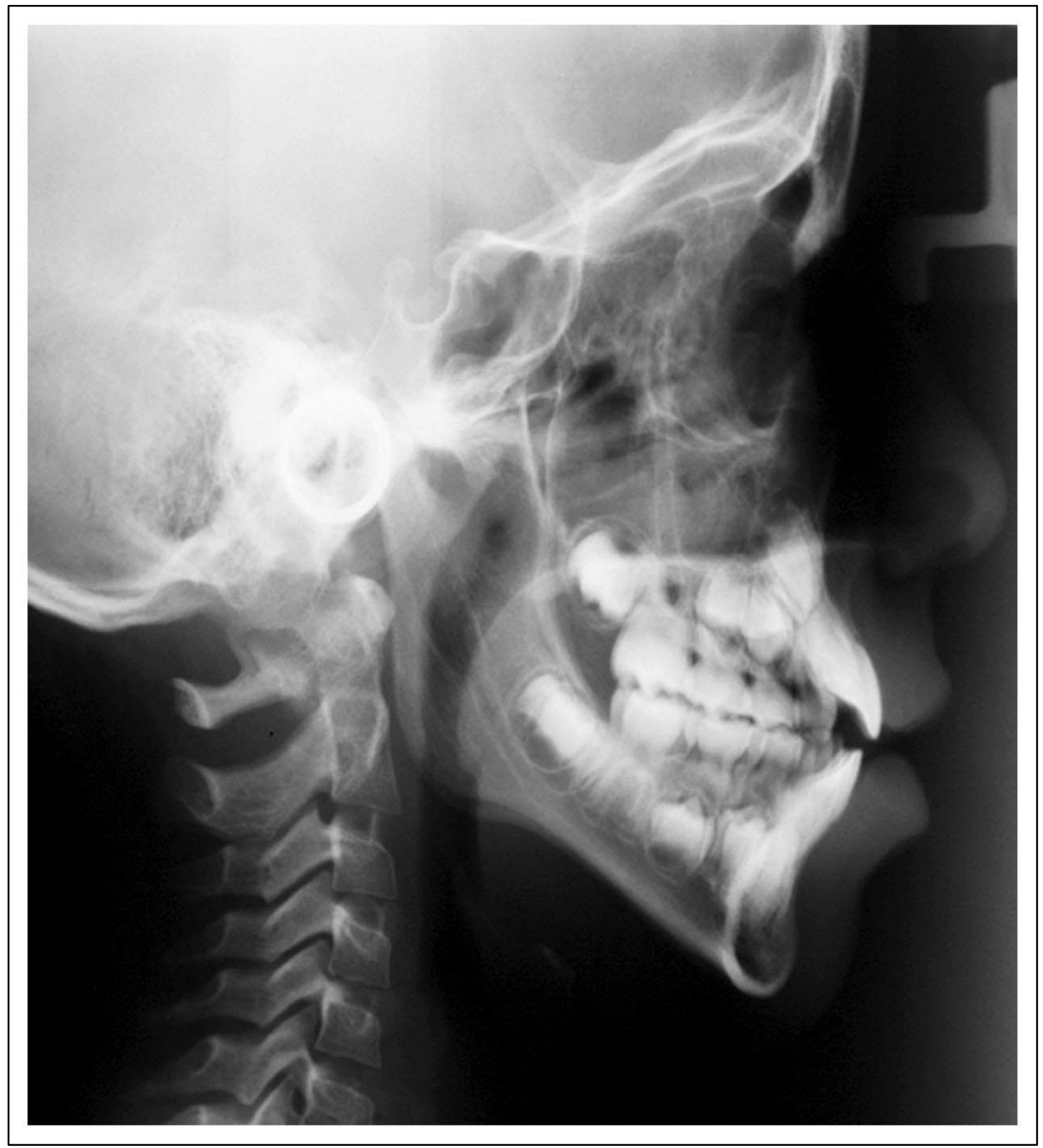

Figura 7 - Radiografia cefalométrica. 


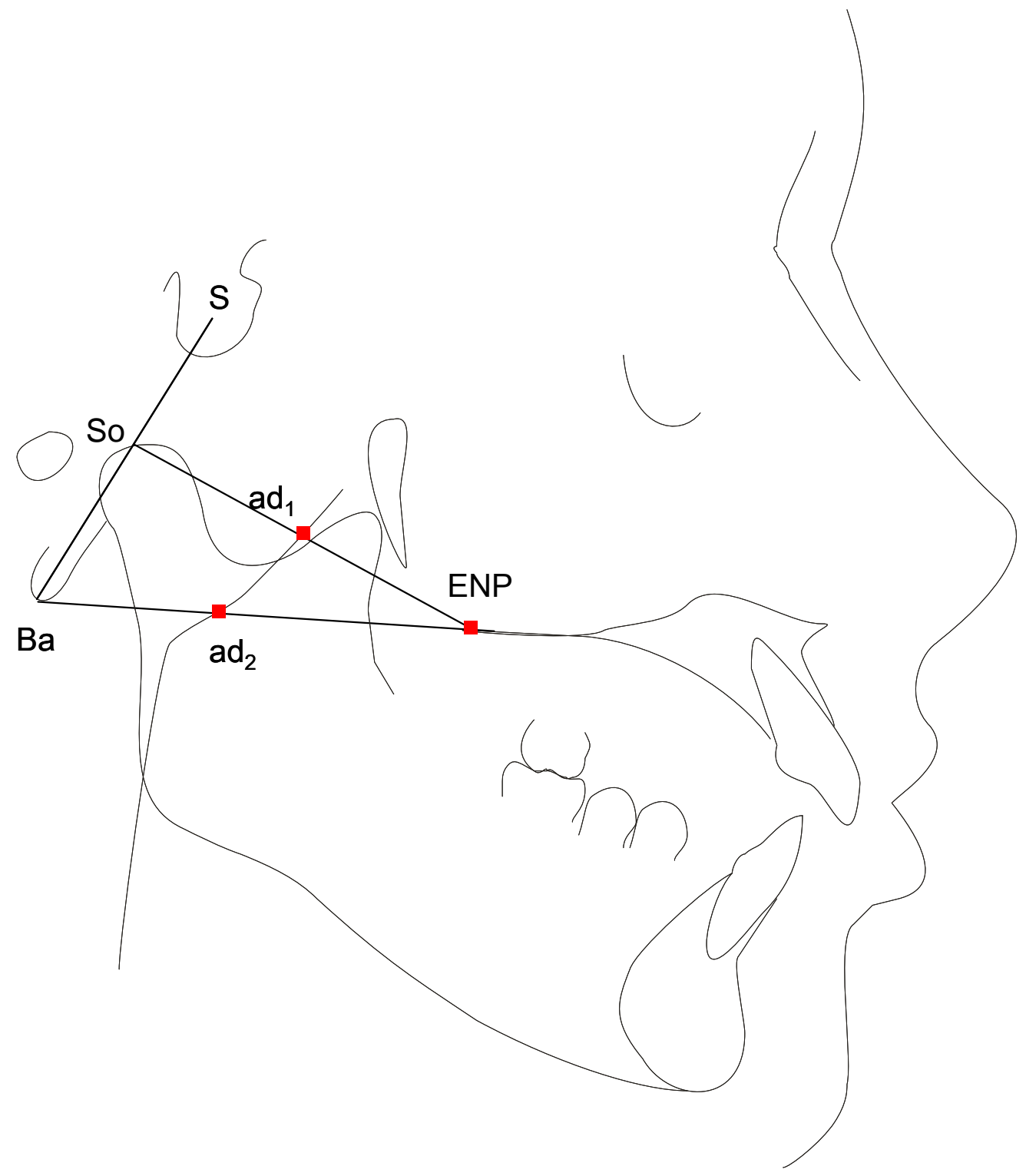

Figura 8 - Traçado cefalométrico em norma lateral, pontos cefalométricos e área da nasofaringe. 


\subsection{RESSONÂNCIA MAGNÉTICA}

Os exames de ressonância magnética (IRM) das vias aéreas superiores, indicados para a avaliação dos efeitos do disjuntor de Haas sobre a região nasofaríngea foram realizados no Centro de Ciências das Imagens e Física Médica do HC/FMRP-USP em equipamento Philips, Achieva de 3.0 T (Tesla), conforme ilustrado na figura 9.

Para cada paciente foi obtida uma série de 160 imagens consecutivas em corte sagital, sendo que o tempo total do exame foi de, aproximadamente, 10 minutos.

Os exames foram realizados antes $\left(T_{1}\right)$ e 6 meses $\left(T_{2}\right)$ após a expansão rápida da maxila.

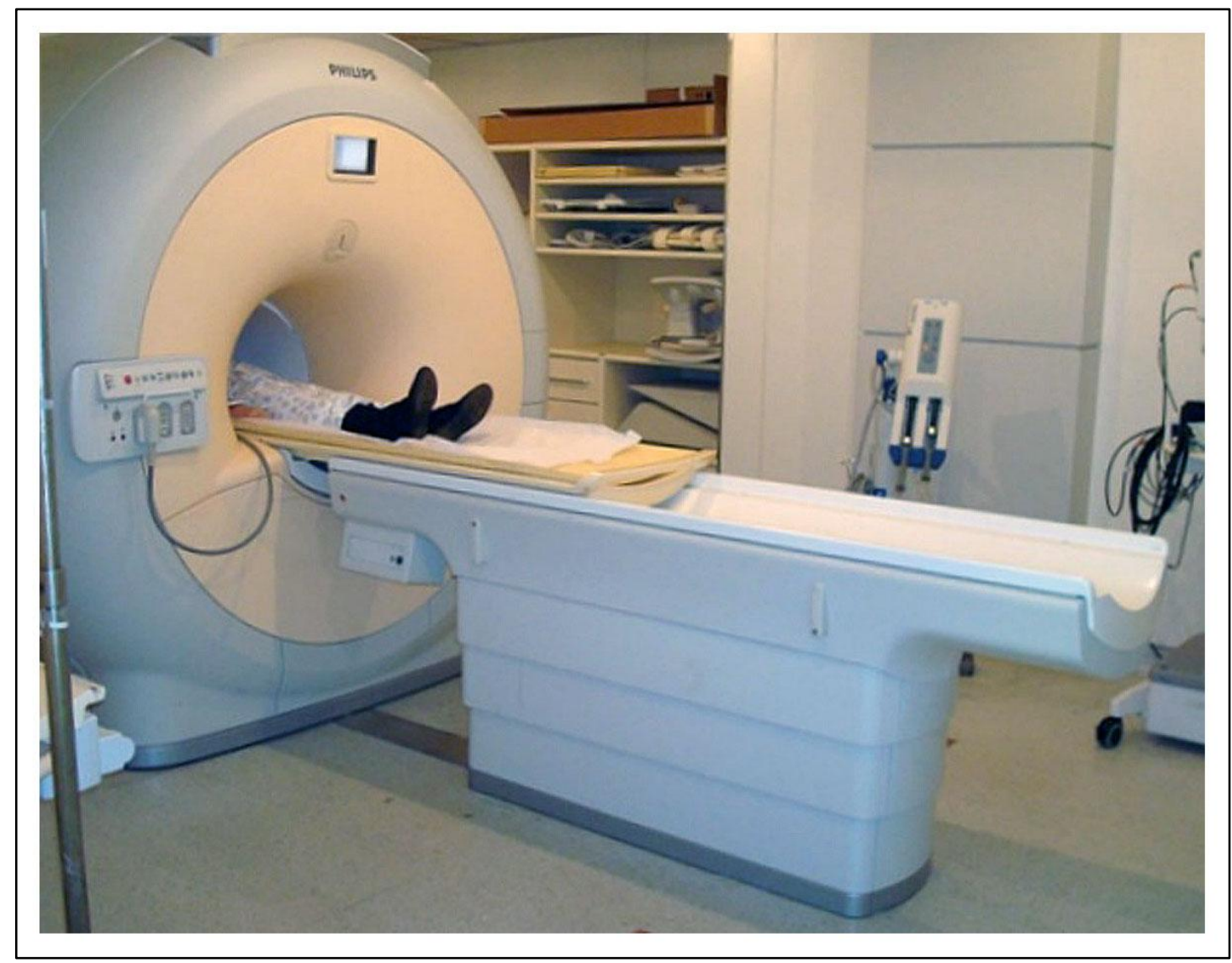

Figura 9: Ressonância magnética: equipamento. 
Após a obtenção das imagens (Figura 10), as mesmas foram transferidas para um computador com o software e Film Workstation 2.1.2, da Merge. Em seguida, sobre cada imagem foi efetuada por meio do mouse a localização dos pontos necessários para a definição da área da nasofaringe. Para isso, utilizou-se o corte sagital mediano ou o mais próximo deste, no qual a hipófise pudesse ser bem visualizada.

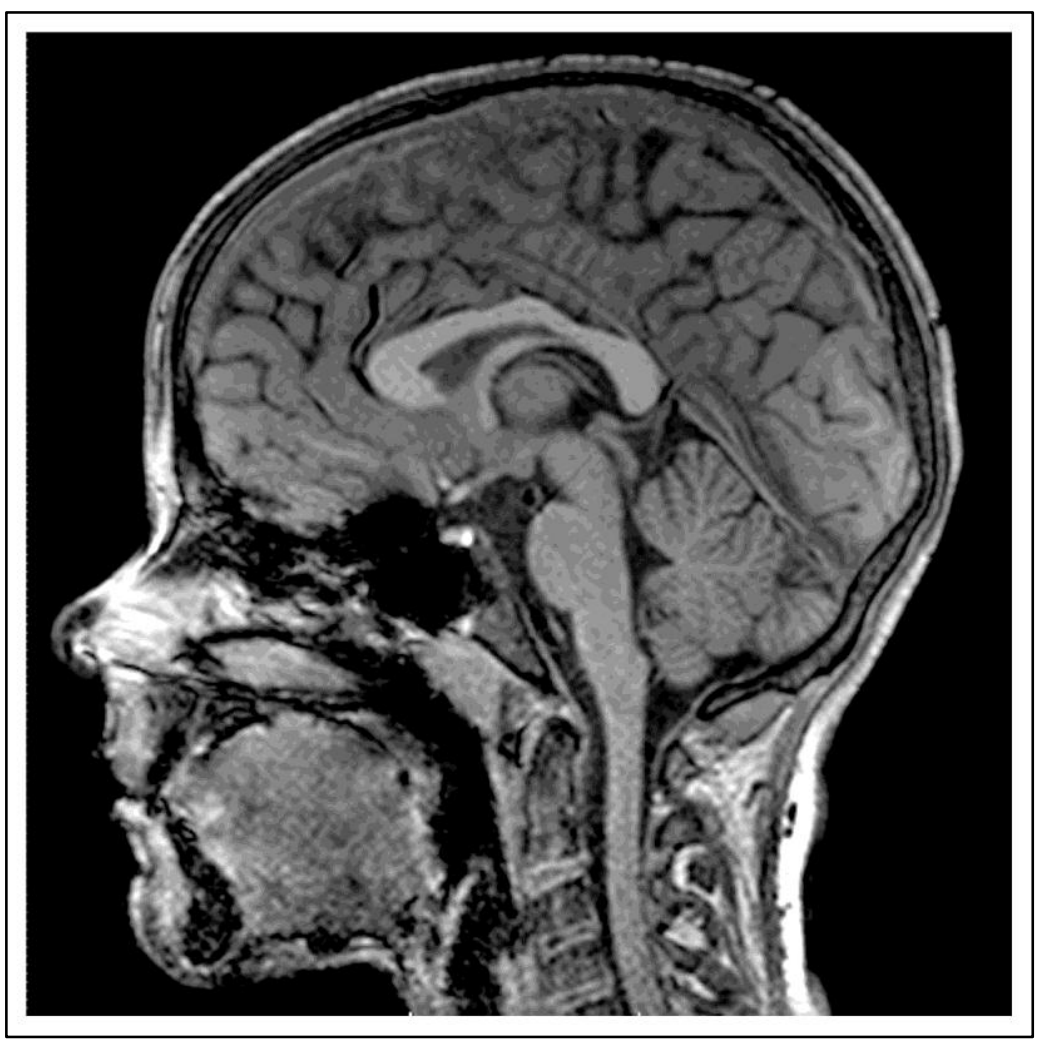

Figura 10 - Imagem de ressonância magnética.

Os parâmetros utilizados por essa técnica para avaliar a área da nasofaringe basearamse nos pontos anatômicos e medidas cefalométricas descritos segundo Linder-Aronson e Henrikson (1973), conforme mostra a figura 8, sendo exatamente os mesmos utilizados na avaliação feita na radiografia cefalométrica lateral.

Devido à dificuldade de visualização da ENP na IRM, optou-se por defini-la como ponto mais anterior do palato mole, ou seja, o limite de transição entre o tecido do palato mole e a gordura da medula óssea do palato duro. Da mesma forma, o centro da sela turca foi 
definido como o ponto mais central da glândula hipófise, que se encontra alojada no seu interior.

A área da nasofaringe visualizada na IRM pode ser observada na figura 11 . As imagens foram avaliadas por dois observadores, simultaneamente, e as medidas da área da nasofaringe, calculadas em $\mathrm{cm}^{2}$, foram obtidas pelo consenso entre eles. Vale ressaltar que os observadores foram previamente testados na avaliação das medidas da área por um profissional da área de radiologia. Finalmente, em nove casos foram realizadas novas medidas após 15 dias da primeira mensuração e, quando a reprodutibilidade foi testada através do Coeficiente de Correlação Intraclasse para verificar o grau de concordância intraobservadores, este apresentou um valor igual a 0,89, ou seja, uma concordância excelente.

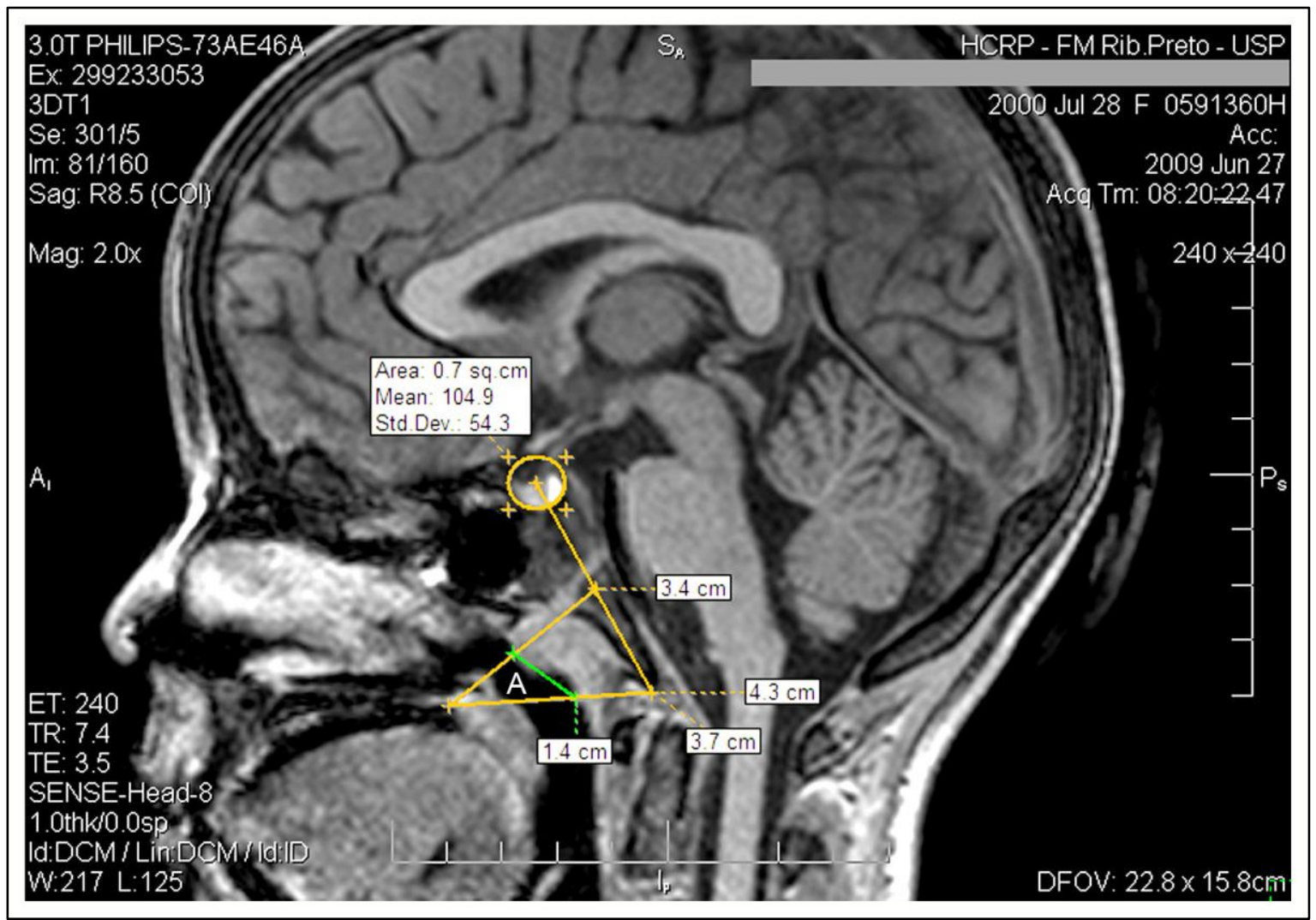

Figura 11 - Área da nasofaringe em imagem de ressonância magnética (A). 
Devido às diferentes datas de realização dos exames tanto de IRM como da radiografia cefalométrica lateral antes da expansão rápida da maxila $\left(T_{1}\right)$ entre todas as crianças estudadas, buscou-se possível influência desse fator sobre as medidas da área da nasofaringe. Para isso, estudou-se a diferença entre os momentos ( $\Delta$ meses) do primeiro e do segundo exame de IRM e a diferença entre as medidas da área da nasofaringe ( $\Delta$ área), antes e após a expansão rápida da maxila. O mesmo foi feito para a radiografia cefalométrica lateral. O coeficiente de correlação de Spearman demonstrou haver uma relação de independência entre aquelas diferenças, permitindo, assim, que todas as crianças pudessem ser estudadas simultaneamente.

O método de estudo foi a mensuração da área da nasofaringe utilizando os mesmos pontos anatômicos nas radiografias cefalométricas laterais e nas imagens de ressonância magnética, antes e 6 meses após a expansão rápida da maxila e posterior correlação e comparação dos resultados encontrados.

\subsection{ANÁLISE ESTATÍSTICA}

A análise estatística dos resultados obtidos foi realizada por meio de metodologia descritiva e analítica. Os dados descritivos foram analisados por meio de médias, desvios padrão e valores mínimo e máximo. Para o estudo analítico foi utilizado o teste $t$ de Student para amostras pareadas e o coeficiente de correlação de Spearman. O pacote estatístico SPSS (versão 17.0) foi utilizado para tabulação dos dados. Adotamos como nível de significância $\alpha \leq 0,05$. 
RESULTADOS 


\section{RESULTADOS}

Os resultados do presente estudo foram demonstrados por meio de tabelas e figuras. Os dados foram divididos e analisados descritiva e analiticamente.

Quando do estudo analítico, os testes e o seu nível de significância foram referidos.

\subsection{Estudo descritivo dos resultados}

Do total de 30 crianças, 16 (53,33\%) eram do gênero masculino e 14 (46,67\%) do feminino. A média de idade entre os meninos foi de $8,4 \pm 1,2$ anos e, entre as meninas, de $8,2 \pm 0,7$ anos, sendo que entre os meninos, a idade mínima foi de 7 anos e a máxima de $10 \mathrm{a} 7 \mathrm{~m}$, e entre as meninas, de $7 \mathrm{a} 3 \mathrm{~m}$ e $9 \mathrm{a} 5 \mathrm{~m}$, respectivamente.

Dos meninos, 12 eram leucodermas e 4 melanodermas; dentre as meninas, 8 eram leucodermas e 6 eram melanodermas.

A nasofibroscopia mostrou que 9 pacientes (30,0\%) apresentaram de 10 a $30 \%$ de obstrução do cavum; 7 (23,3\%) apresentaram de 30 a 50\%; 4 pacientes (13,3\%), de 50 a $70 \%$ e $10(33,3 \%)$ apresentaram mais de $70 \%$ de obstrução.

Os dados referentes aos valores das medidas da área da nasofaringe, obtidas por meio do exame de ressonância magnética e por meio da radiografia cefalométrica lateral, antes $\left(T_{1}\right)$ e após $\left(T_{2}\right)$ a expansão rápida da maxila, encontram-se na tabela 1. 
Tabela 1 - Médias, desvios padrão (DP), valores mínimo (Mín) e máximo (Máx) das medidas da área da nasofaringe obtidas pela ressonância magnética e pela radiografia cefalométrica lateral, realizadas nos momentos $\mathrm{T}_{1}$ e $\mathrm{T}_{2}$

\begin{tabular}{ccccccc}
\hline Exames & Tempos & Média & DP & Mín & Máx & Valor de $p$ \\
\hline IRM & $\mathrm{T}_{1}$ & 0,73 & 0,29 & 0,20 & 1,53 & $\mathrm{P}<0,001^{*}$ \\
& $\mathrm{~T}_{2}$ & 0,98 & 0,41 & 0,36 & 1,96 & \\
Cef & $\mathrm{T}_{1}$ & 0,66 & 0,34 & 0,05 & 1,37 & $\mathrm{P}=0,21 \mathrm{NS}$ \\
& $\mathrm{T}_{2}$ & 0,71 & 0,36 & 0,07 & 1,60 & \\
\hline
\end{tabular}

IRM=ressonância magnética; $T_{1}=$ antes da $E R M ; T_{2}=180$ dias após a ERM; Cef=radiografia cefalométrica lateral; Valor de $p={ }^{*}$ estatisticamente significante $(p<0,05)$ e ns- não significante $(p>0,05)$.

\subsection{Estudo analítico dos resultados}

\subsubsection{Radiografia cefalométrica lateral}

A análise estatística utilizando o teste $t$ de Student para amostras pareadas mostrou que não houve diferença estatisticamente significante $(p=0,21)$ entre a área da nasofaringe, antes e após a expansão rápida da maxila (ERM), conforme demonstra a figura 12 e tabela 1.

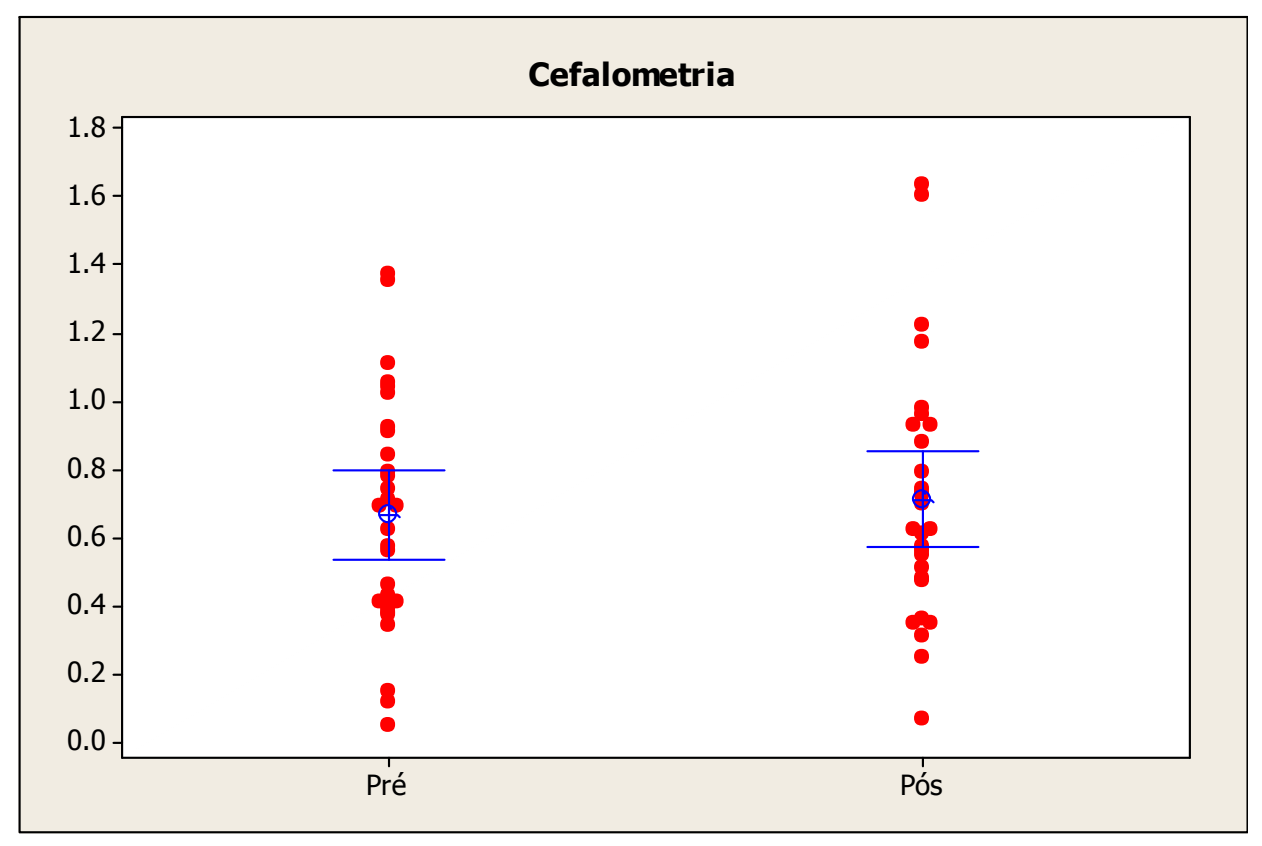

Figura 12 - Média da área da nasofaringe das crianças submetidas à radiografia cefalométrica lateral, antes e após a ERM. 


\subsubsection{Ressonância magnética}

A análise estatística utilizando o teste $t$ de Student para amostras pareadas mostrou que houve diferença estatisticamente significante $(p<0,001)$ entre a área da nasofaringe, antes e após a expansão rápida da maxila (ERM), conforme demonstra a figura 13 e tabela 1.

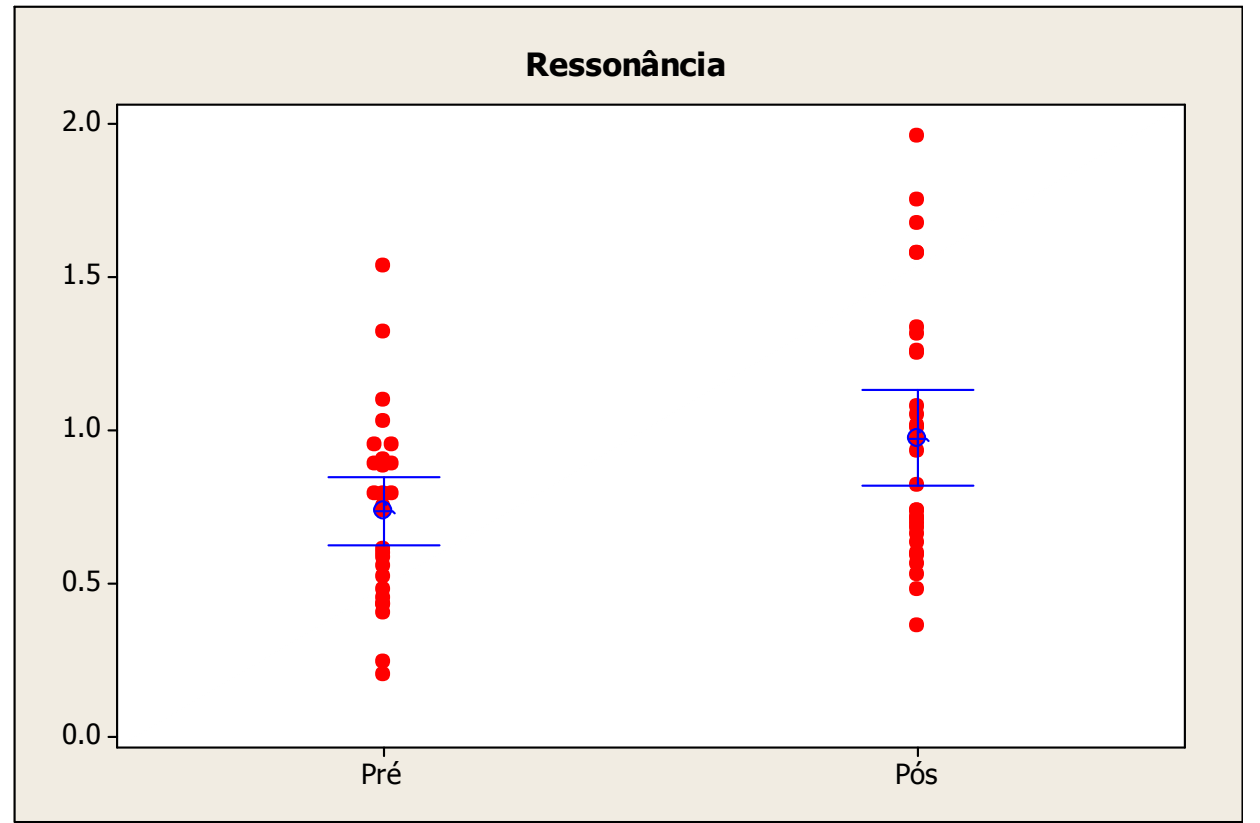

Figura 13 - Média da área da nasofaringe das crianças submetidas à ressonância magnética, antes e após a ERM.

\subsubsection{Correlação e comparação entre a área da nasofaringe pré e pós ERM por meio dos exames de ressonância magnética e radiografia cefalométrica lateral}

Houve correlação positiva entre a área da nasofaringe medida por meio da ressonância magnética (IRM) e da radiografia cefalométrica lateral, antes ( $p=0,86$; Figura 14) e após ( $p=0,81$; Figura 15) a expansão rápida da maxila (ERM), quando analisada pelo coeficiente de correlação de Spearman. Quando foi feita a comparação entre esses métodos por meio do teste $t$ de Student para amostras pareadas (Tabela 2), observou-se diferença estatisticamente significante entre os valores da radiografia cefalométrica lateral e os da ressonância magnética antes (Figura 16) e após (Figura 17) a ERM, sendo os valores da ressonância significativamente maiores aos da radiografia cefalométrica. 


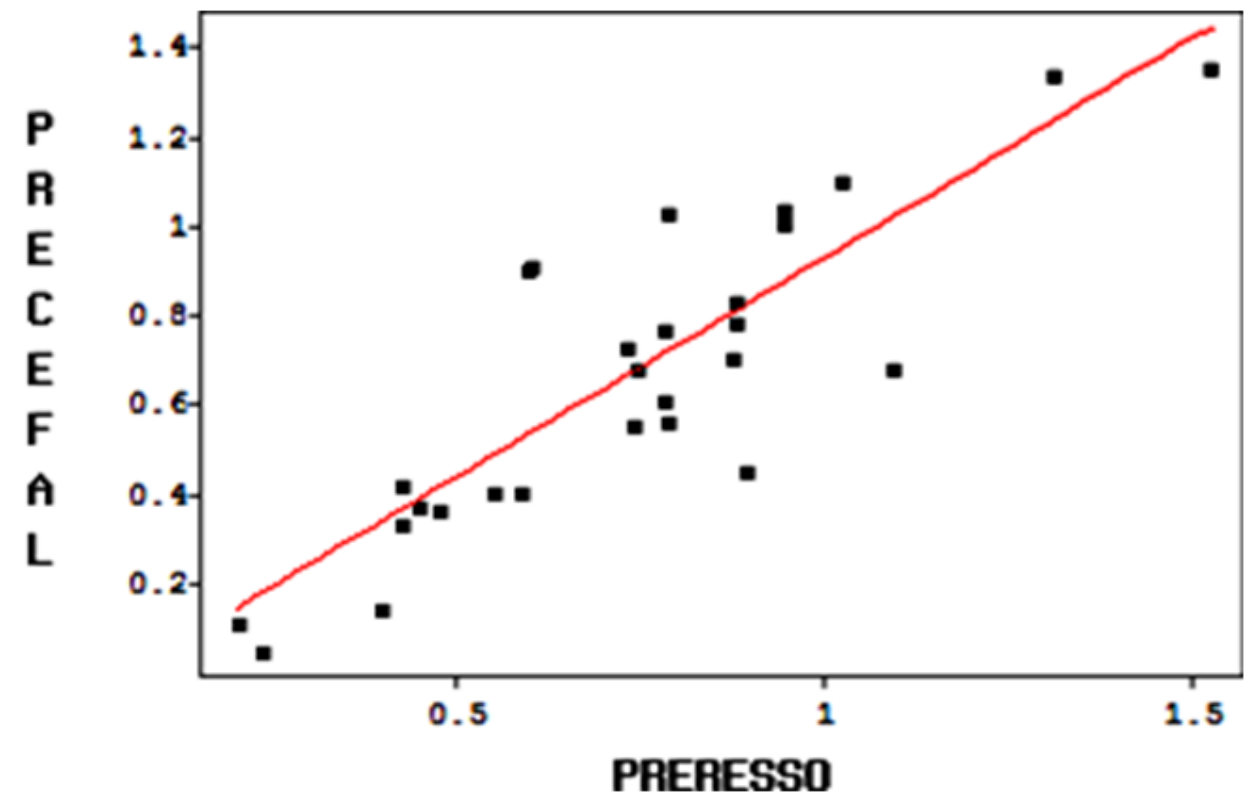

Figura 14 - Correlação entre a área da nasofaringe por meio da IRM e radiografia cefalométrica lateral antes da ERM.

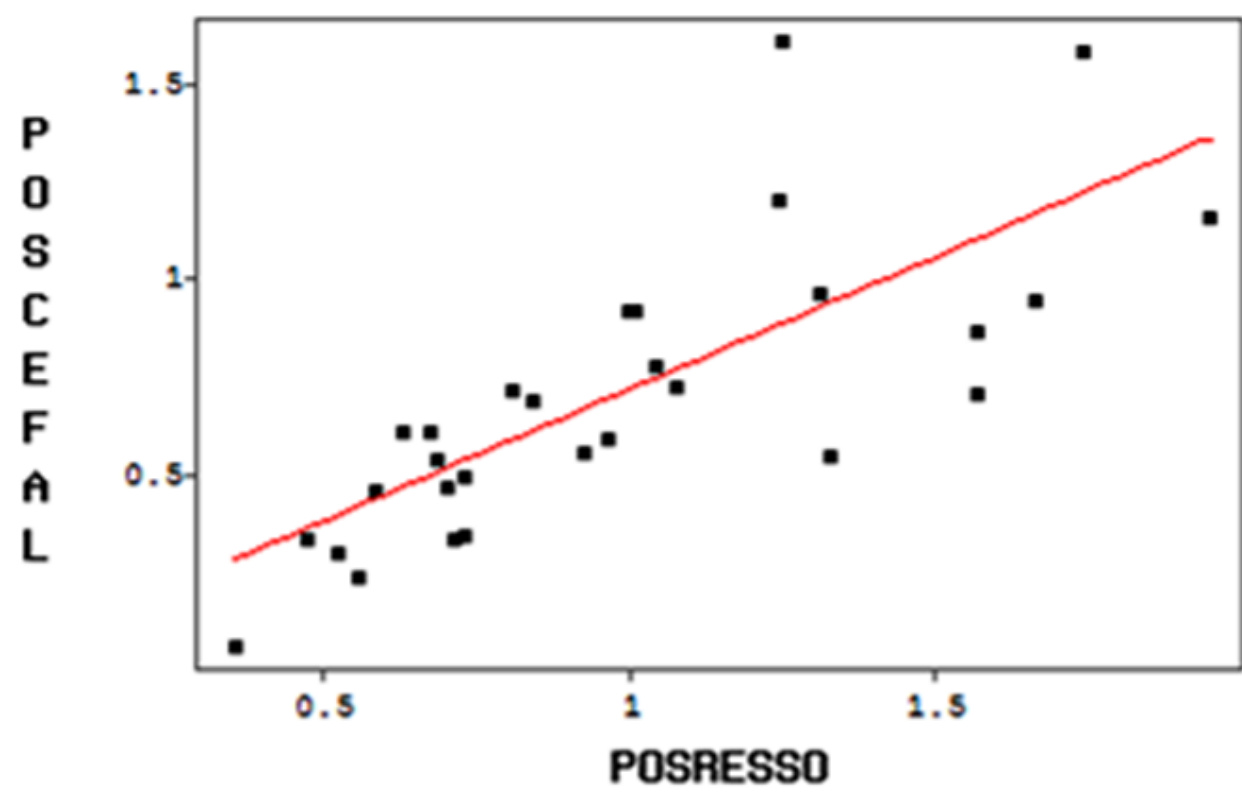

Figura 15 - Correlação entre a área da nasofaringe por meio da IRM e radiografia cefalométrica lateral após a ERM. 
Tabela 2 - Comparação das medidas da área da nasofaringe obtidas pela ressonância magnética e pela radiografia cefalométrica lateral, realizadas nos momentos $T_{1}$ e $T_{2}$

\begin{tabular}{ccccc}
\hline Tempos & Exames & Média & DP & Valor de $p$ \\
\hline $\mathrm{T}_{1}$ & IRM & 0,73 & 0,29 & $\mathrm{P}=0,04^{*}$ \\
& Cef & 0,66 & 0,34 & \\
$\mathrm{~T}_{2}$ & IRM & 0,98 & 0,41 & $\mathrm{P}<0,001^{*}$ \\
& Cef & 0,71 & 0,36 & \\
\hline
\end{tabular}

IRM=ressonância magnética; $\mathrm{T}_{1}=$ =antes da ERM; $\mathrm{T}_{2}=180$ dias após a ERM; Cef=radiografia cefalométrica lateral.

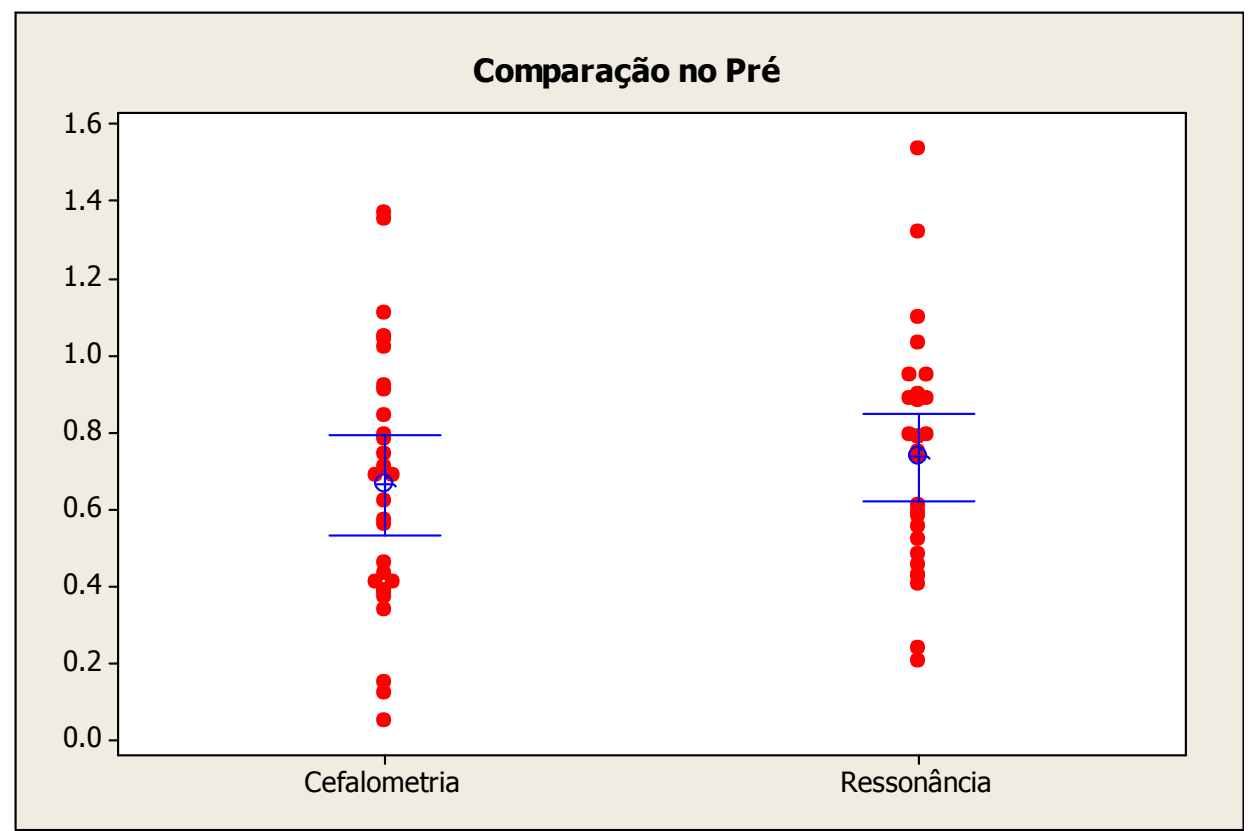

Figura 16 - Comparação entre as medidas da área da nasofaringe por meio da radiografia cefalométrica lateral e da ressonância magnética antes da ERM.

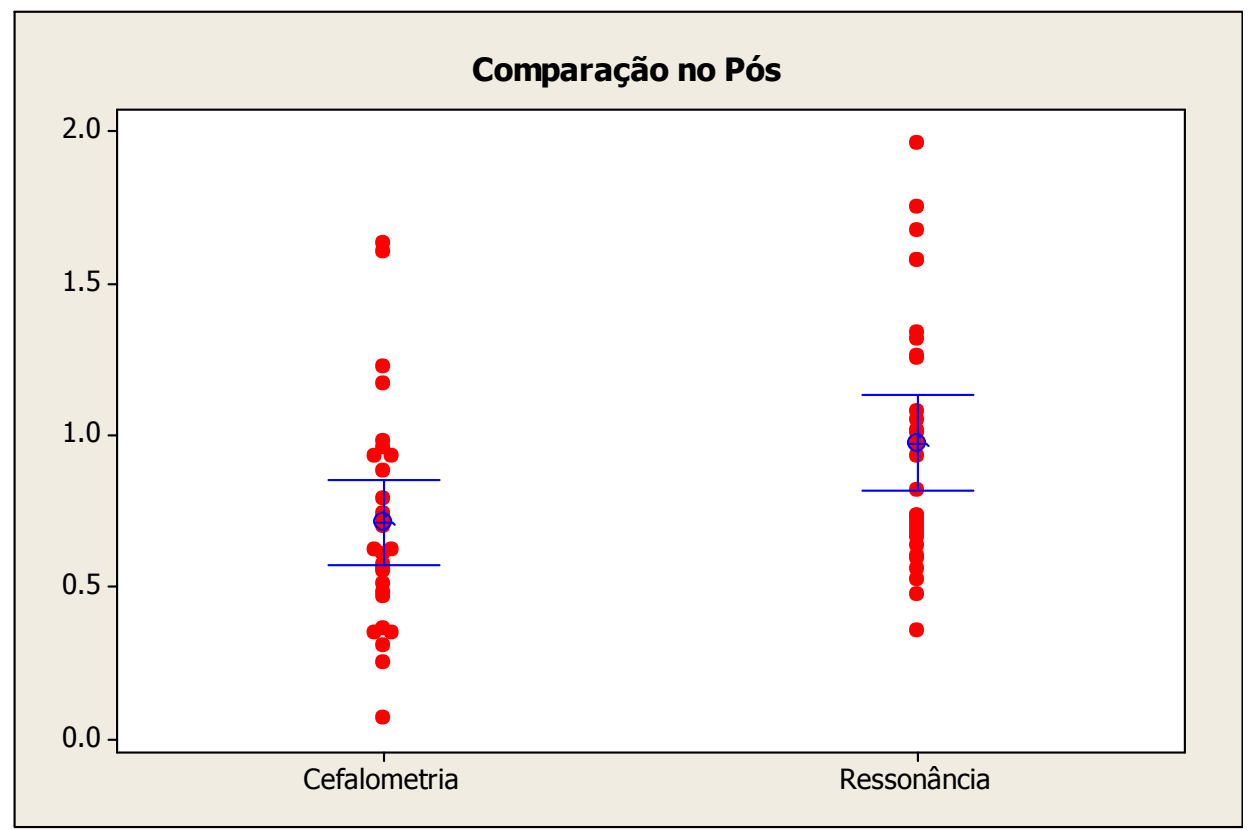

Figura 17 - Comparação entre as medidas da área da nasofaringe por meio da radiografia cefalométrica lateral e da ressonância magnética 180 dias após a ERM. 
Discussão 


\section{DISCUSSÃO}

$\mathrm{Na}$ literatura a constrição maxilar tem sido associada a alterações morfológicas da parte anterior da cavidade nasal assim como do espaço nasofaríngeo (Haas, 1961; Monini et al., 2009). Vários estudos clínicos têm demonstrado aumento significante nas dimensões transversais da maxila e cavidade nasal (Basciftci et al., 2002; Chung e Font, 2004; Lagravere et al., 2005), diminuição na resistência aérea nasal, aumento no espaço nasofaríngeo e melhora na respiração (Da Silva Filho et al., 1995; Basciftci et al., 2002; Enoki et al., 2006; Ramires et al., 2008; Monini et al., 2009; Matsumoto et al., 2010; Langer et al., 2011) após a ERM realizada para correção da mordida cruzada posterior.

O objetivo deste estudo foi avaliar o efeito da ERM sobre as dimensões do espaço nasofaríngeo por meio de radiografia cefalométrica lateral e imagem de ressonância magnética, nas quais foram utilizados os pontos anatômicos e as medidas cefalométricas descritas por Linder- Aronson e Henrikson (1973). A amostra deste estudo foi composta por 30 crianças, de ambos os gêneros, na faixa etária de 7 a 10 anos, com diagnóstico de respiração bucal e/ou mista e mordida cruzada posterior uni ou bilateral e que tinham a indicação de realizar a ERM. O disjuntor de Haas foi utilizado para correção da maloclusão e os exames de imagem foram realizados antes da instalação do aparelho e 6 meses após a sua estabilização.

Os métodos usados para avaliar as dimensões do espaço nasofaríngeo em práticas ortodônticas são limitados (Pirila-Parkkinen et al., 2011), sendo que os mais utilizados na literatura para essa avaliação são as radiografias cefalométricas laterais e mais recentemente as tomografias computadorizadas. Considerando que a imagem de ressonância magnética não apresenta radiação ionizante, e associada ao fato desta pesquisa ser realizada em um centro multidisciplinar com possibilidade de acesso a este exame, optamos por complementar o trabalho incluindo-o na metodologia estudada.

Os resultados encontrados no presente estudo mostraram que houve aumento no espaço nasofaríngeo 6 meses após a ERM quando analisado na radiografia cefalométrica lateral e na ressonância magnética. Entretanto, na IRM este aumento foi mais expressivo, sendo estatisticamente significante, enquanto que o aumento encontrado na RCL não atingiu significância estatística. 
Esse resultado constatado no presente estudo, ao comparar a área nasofaríngea por meio de RCL e IRM, pode ser devido ao fato de que a IRM possibilita obter imagens fidedignas dos tecidos moles, porém, dificulta a visualização de limites ósseos. Pode-se citar como exemplo, a dificuldade de identificação da ENP e o centro da sela turca na IRM. Entretanto, essa dificuldade foi superada, pois a ENP foi definida como o ponto mais anterior do palato mole, ou seja, o limite da transição entre o tecido do palato mole e a gordura da medula óssea do palato duro, conforme descreveram Welch et al. (2002). Este é o ponto mais anterior do palato mole e melhor identificável na IRM. Da mesma forma, o centro da sela turca foi definido como o ponto mais central da glândula hipófise, a qual se encontra alojada no seu interior. Os pontos $\operatorname{ad}_{1}$ e $\operatorname{ad}_{2}$ que são definidos a partir do contorno do tecido linfóide adenoideano, têm melhor definição na IRM, uma vez que, há um consenso na literatura de que a IRM reproduz com mais precisão as imagens de tecidos moles, como palato mole, língua e paredes laterais da faringe. Por outro lado, as imagens obtidas por meio da RCL não têm a mesma precisão, pois sofrem distorções devido às características peculiares à técnica de obtenção dessas radiografias.

Em concordância com os resultados obtidos neste estudo, Picchi et al. (1990) não observaram alteração significativa nas dimensões do espaço nasofaríngeo após ERM, por meio da cefalometria.

Kurt et al. (2010) também não constataram diferenças estatisticamente significantes nas dimensões do espaço nasofaríngeo após ERM. Entretanto, esses autores avaliaram 10 pacientes com idade variando entre 13 e 17 anos, com média de 15 anos e o aparelho utilizado foi o Hyrax. Do mesmo modo, Langer et al. (2011) não encontraram diferenças significativas na área da nasofaringe avaliada por meio de radiografia cefalométrica após ERM, de 25 crianças respiradoras bucais de 7 a 10 anos, com MCP uni ou bilateral

Em contrapartida, autores como Basciftci et al. (2002), Buccheri et al. (2004), Chiari et al. (2009) e Monini et al. (2009) que também utilizaram radiografia cefalométrica lateral para avaliar os efeitos da ERM nas dimensões do espaço nasofaríngeo observaram aumento significativo desse espaço. Basciftci et al. (2002) constataram aumento significante do espaço nasofaríngeo de crianças de 12 e 14 anos, por meio da radiografia cefalométrica lateral, antes e 3 meses após a expansão. Entretanto, a área nasofaríngea (AN) e a área respiratória (AR) foram medidas por meio de um planímetro digital. 
Com resultados semelhantes, Buccheri et al. (2004) verificaram aumento da largura do espaço nasofaríngeo em 24 crianças respiradoras bucais com diagnóstico de hiperplasia adenoideana, porém, mais jovens, entre 5 e 9 anos. A avaliação foi realizada 5 meses após a ERM.

Outros autores como Chiari et al. (2009) investigaram os efeitos da ERM sobre as vias aéreas superiores e o ouvido médio. A análise cefalométrica mostrou que em ambos os grupos houve aumento do espaço nasofaríngeo no plano sagital após 6 meses da ERM, que nesses casos foi realizada com o disjuntor de Hyrax. Entretanto, esses autores avaliaram um grupo de apenas 7 crianças com mordida cruzada posterior uni ou bilateral, com média de 8 anos de idade.

O mesmo resultado foi encontrado no estudo de Monini et al. (2009), cuja análise cefalométrica mostrou aumento significativo do espaço nasofaríngeo 1 ano após a ERM. A amostra desse estudo constou de 65 crianças, entre 5 e 10 anos, que apresentavam constrição maxilar e obstrução nasal por diversas causas. No entanto, em somente 13 crianças foram realizadas as radiografias cefalométricas laterais com objetivo de avaliar o espaço nasofaríngeo.

Portanto, Buccheri et al. (2004), Chiari et al. (2009), Monini et al. (2009) estudaram crianças na mesma faixa etária do presente estudo, porém, Buccheri et al. (2004) selecionaram somente crianças com hiperplasia de adenóide e a análise cefalométrica foi realizada 5 meses pós tratamento. Monini et al. (2009) reavaliaram o espaço nasofaríngeo de 13 crianças submetidas à ERM, 1 ano após a estabilização do aparelho, tempo superior à nossa pesquisa. Chiari et al. (2009) realizaram as análises cefalométricas no mesmo intervalo de tempo do presente estudo, 6 meses após ERM, porém, a amostra era composta de apenas 7 crianças e o aparelho utilizado foi o disjuntor de Hyrax, que tem efeito similar ao disjuntor de Haas utilizado no presente trabalho.

O aumento da área da nasofaringe deve-se provavelmente ao deslocamento anterior e inferior da maxila após a ERM (Haas, 1961), uma vez que o intervalo de tempo de 6 meses pós-disjunção, em crianças na faixa etária de 7 a 10 anos, antes do surto de crescimento puberal, seria insuficiente para promover o crescimento da nasofaringe (Vilella et al., 2006). No estudo de Chung e Font (2004) os autores observaram que 3 meses após a expansão houve um deslocamento anterior da maxila de aproximadamente 0,58 $\mathrm{mm}$, concordando com os achados de Akkaya et al. (1999). Contudo, essa movimentação não é consenso na 
literatura, pois autores como Da Silva Filho et al. (1991) e Capelozza Filho et al. (1994) observaram apenas o deslocamento inferior da maxila, ao avaliar 32 crianças, de 5 a 11 anos, com constrição maxilar após ERM. Outra possível explicação para esse achado, diz respeito ao aumento de aproximadamente $1 \mathrm{~mm}$ na distância entre os processos pterigóides do osso esfenóide após a ERM (Lione et al., 2008), pois, a parte superior da faringe está conectada a essas estruturas, podendo influenciar no ganho da área da nasofaringe (Buccheri et al., 2004).

Os resultados do presente estudo demonstraram correlação positiva $(p<0,001)$ entre a área da nasofaringe medida por meio da ressonância magnética e da radiografia cefalométrica lateral nos dois momentos de realização destes exames, antes e após a expansão rápida da maxila (ERM), ou seja, houve aumento da área nasofaringea após a ERM, o que corrobora com os achados de Pirila-Parkkinen et al. (2011) que avaliaram crianças com apnéia.

Lenza et al. (2010) avaliaram a correlação de medidas lineares (sagital e transversal), áreas e volumes da via aérea superior determinadas em radiografias cefalométricas laterais e tomografia computadorizada tipo "cone beam" (TCCB). Em relação à porção nasofaringeana da via aérea superior, somente a parede posterior superior da nasofaringe $\left(\operatorname{ad}_{2}-\mathrm{ENP}\right)$ teve correlação alta com a área e a expressão de volume da via aérea nesta região. Observaram também correlação positiva entre as áreas transversais da nasofaringe inferior e velofaringe superior e seus volumes parciais correspondentes. Este achado está em concordância com Aboudara et al. (2009), que reportaram correlação positiva entre a medida do tamanho da via aérea nasofaríngea em radiografias cefalométricas laterais e seu volume real calculado por meio de TCCB. Para a porção inferior da via aérea superior, foi observada correlação fraca entre todos os parâmetros, o que está de acordo com Vig e Hall (1980), que questionaram o uso da radiografia cefalométrica lateral para avaliação da morfologia total da via aérea superior, pelo fato das medidas sagitais dessas radiografias não poderem antecipar a morfologia da via aérea superior, exceto para a parte da nasofaringe.

Os trabalhos acima citados concordam com os resultados da nasofaringe encontrados neste estudo. Entretanto, Aboudara et al. (2009) e Lenza et al. (2010) compararam as radiografias cefalométricas laterais com exames de TCCB e Vig e Hall (1980) utilizaram apenas radiografias cefalométricas laterais. 
Segundo revisão sistemática da literatura feita por Major et al. (2006) os diagnósticos a cerca de obstruções das vias aéreas posteriores, como a nasofaringe, por meio de radiografias cefalométricas ainda apresentam deficiências sérias, como tamanho das amostras, fatores de idade e falta de um rigoroso padrão. Esses exames se mostraram métodos úteis no diagnóstico do tamanho da adenóide, melhor que no diagnóstico do tamanho da nasofaringe (Major et al., 2006; Pirila-Parkkinen et al., 2011).

A partir dos resultados apresentados, a radiografia cefalométrica lateral que apresenta uma avaliação bidimensional não se mostrou totalmente precisa na mensuração de estruturas complexas tridimensionais como a nasofaringe, o que corrobora com os achados de Lenza et al. (2010). Dessa forma, recomenda-se uma análise volumétrica tridimensional por meio da IRM, que oferece melhor resolução dos tecidos moles comparada com outras técnicas rotineiramente utilizadas para avaliar as estruturas das vias aéreas superiores (Arens et al., 2001 e 2002). A IRM fornece dados precisos sobre as características e mensurações da nasofaringe, variações no tamanho das tonsilas palatinas e faríngeas, incluindo tecido adiposo, assim como de todas as estruturas das vias aéreas superiores, o que pode melhorar o diagnóstico e ajudar a localizar eventuais obstáculos nessa região, o que consequentemente afetará o padrão respiratório normal (Songu et al., 2010). A IRM determina com exatidão o volume e a área transversal, permitindo imagens no plano axial, sagital e coronal com reconstruções volumétricas tridimensionais de tecidos moles e estruturas ósseas. Portanto, sugere-se estudos complementares utilizando análise tridimensional obtida por meio de IRM.

Desta forma, pode-se afirmar que os dois métodos de avaliação utilizados para mensurar a dimensão nasofaríngea têm suas indicações, pois a radiografia cefalométrica lateral além de ser mais acessível e estar disponível na maioria dos centros de diagnóstico, tem custo financeiro muito menor que a ressonância magnética, que mesmo sendo um exame preciso e de imagens de alta resolução dos tecidos da via aérea superior, ainda não está ao alcance da maioria da população. 
CONCLUSÃO 


\section{CONCLUSÃO}

6.1 Com relação ao efeito da expansão rápida da maxila sobre a dimensão do espaço nasofaríngeo:

6.1.1 Não houve alteração significativa na dimensão do espaço nasofaríngeo após a ERM, avaliado por meio da radiografia cefalométrica lateral;

6.1.2 Houve aumento significativo do espaço nasofaríngeo após ERM, avaliado por meio da IRM;

6.2 Quanto à comparação e correlação dos dados obtidos nos dois exames de imagem:

6.2.1 Houve correlação positiva entre os valores obtidos nos dois exames;

6.2.2 Houve diferença estatisticamente significante quando comparados os valores obtidos nos dois exames.

Os dois métodos de medição utilizados para avaliar a dimensão nasofaríngea apresentaram limitações, pois, a radiografia cefalométrica lateral fornece uma visão bidimensional da área e a IRM não foi explorada para se obter uma visão volumétrica tridimensional. 
REFERÊNCIAS 


\section{REFERÊNCIAS}

1- Aboudara CA, Nielsen I, Huang JC et al. Comparision of airway space with conventional lateral headfilms and 3-dimensional reconstruction of cone beam computed tomography in airway imaging: a review. Seminars in Orthodontics 2009;15:63-9.

2- Akkaya S, Lorenzon S, Uçem TT. A comparison of sagittal and vertical effects between bonded rapid and slow maxillary expansion procedures. Eur J Orthod 1999;21(2):175-180.

3- Arens R, McDonough JM, Costarino AT. Magnetic resonance imaging of the upper airway structure of children with obstructive sleep apnea syndrome. Am J Respir Crit Care Med 2001; 164:698-703.

4- Arens $\mathrm{R}, \mathrm{McD}$ otiough JM, Corbin AM. Linear dimensions of the upper airway structure during development: assessment by magnetic resonance imaging. Am J Respir Crit Care Med 2002; 165:11722.

5- Bahadir O. Effects of adenoidectomy in children with symptoms of adenoidal hypertrophy. Eur Arch Otorhinolaryngol 2006; 263(2): 156-9.

6- Baik UB, Suzuki M, Ikeda K, Sugawara J, Mitani H. Relationship between cephalometric characteristics and obstructive sites in obstructive sleep apnea syndrome. Angle Orthod 2002;72(2):124-134.

7- Ballanti F, Lione R, Baccetti T, Lorenzo F, Cozza P. Treatment and posttreatment skeletal effects of rapid maxillary expansion investigated with low-dose computed tomography in growing subjects. Am J Orthod Dentofacial Orthop 2010;138:311-7.

8- Basciftci FA, Mutlu N, Karaman Al, Malkoc S, Küçükkolbasi H. Does the timing and method of rapid maxillary expansion have an effect on the changes in nasal dimensions? Angle Orthod 2002;72(2):118-23.

9- Bishara SE, Stanley RN. Maxillary expansion: clinical implications. Am J Orthod Dentofacial Orthop 1987;91:3-14.

10- Branco A, Ferrari GF, Weber SAT. Alterações orofaciais em doenças alérgicas de vias aéreas. Rev Paul Pediatr 2007;25(3):266-270.

11- Buccheri A, Dilella G, Stella R. Rapid palatal expansion and pharyngeal space. Cephalometric evaluation. Prog Orthod 2004; 5(2):160-71. 
12- Capelozza Filho L, Mazzottini R, Cardoso Neto J, Silva Filho OG. Expansão rápida da maxila cirurgicamente assistida. Ortodontia 1994;27(1):21-30.

13- Capelozza Filho L, Silva Filho OG. Expansão rápida da maxila: considerações gerais e aplicação clínica. Parte I. Dental Press Ortod Ortop Maxilar 1997;2(3):88-102.

14- Carvalho LV. Má oclusão de dimensão vertical facial excessiva em respiradores bucais: etiologia e diagnóstico. In: Ortodontia e ortopedia facial. Associação Brasileira de Odontologia de Minas Gerais: Belo Horizonte; 2003. p. 111.

15- Chiari S, Romsdorfer P, Swoboda H, Bantleon HP, Freudenthaler J. Effects of rapid maxillary expansion on the airways and ears - a pilot study. Eur J Orthod 2009;31(2):135-41.

16- Chung $\mathrm{CH}$, Font $\mathrm{B}$. Skeletal and dental changes in the sagittal, vertical and transverse dimensions after rapid palatal expansion. Am J Orthod Dentofacial Orthop 2004;126(5):569-75.

17- Cleylan I, Oktay H, Demirci M. The effect of rapid maxillary expansion on conductive hearing loss. Angle Orthod 1996;66(4):301-30.

18- Crouse U, Laine-Alava MT, Warren DW. Nasal impairment in prepuberal children. Am J Orthod Dentofacial Orthop 2000;118(1):69-74.

19- Da Silva Filho OG, Boas MC, Capelozza Filho L. Rapid maxillary expansion in the primary and mixed dentitions: a cephalometric evaluation. Am J Orthod Dentofacial Orthop 1991;100(2):171-79.

20- Da Silva Filho OG, Montes LA, Torelly LF. Rapid maxillary expansion in the deciduous and mixed dentition evaluated through postero-anterior cephalometric analysis. Am J Orthod Dentofacial Orthop 1995;107(3):268-275.

21- Da Silva Filho OG, Lara TS, da Silva HC, Bertoz FA. Post-expansion evaluation of the midpalatal suture in children submitted to rapid palatal expansion: a CT study. J Clin Pediatr Dent 2006;31:1428.

22- Donnelly LF, Casper KA, Chen B. Correlation on cine MR imaging of size of adenoid and palatine tonsils with degree of upper airway motion in asymptomatic sedated children. Am J Roentgenol 2002a;179(2):503-8.

23- Enoki C, Valera FCP, Lessa FCR, Elias AM, Matsumoto MAN, Anselmo-Lima WT. Effect of rapid maxillary expansion on the dimension of the nasal cavity and on nasal air resistance. Int J Pediatr Otorhinolaryngol 2006;70(7):1225-1230. 
24- Faria PTM, Ruellas AC, Matsumoto, MAN. Dentofacial morphology of mouth breathing children. Bras Dent J 2002; 13(2):129-132.

25- Franchi L, Baccetti T, Lione R, Fanucci E, Cozza P. Modifications of midpalatal sutural density induced by rapid maxillary expansion: a low-dose computed tomography evaluation. Am J Orthod Dentofacial Orthop 2010;137:486-488.

26- Galarreta FWM. Efeito da expansão rápida da maxila sobre a nasofaringe e o volume nasal: avaliação por ressonância magnética e rinometria acústica [tese]. Ribeirão Preto: Faculdade de Medicina de Ribeirão Preto da Universidade de São Paulo; 2010.

27- Goldsmith JL, Stool SE. George Catlin's concepts on mouth-breathing as presented by Dr. Edward H. Angle. Angle Orthod 1994;64:75-8.

28- Haas AJ. Rapid Expansion of the maxillary dental arch and nasal cavity by opening the midpalatal suture. Angle Orthod 1961;31(2):73-90.

29- Haas AJ. The treatment of maxillary deficiency by opening the midpalatal suture. Angle Orthod 1965;35:200-217.

30- Jaw TS, Sheu RS, Liu GC, Lin WC. Development of adenoids: a study by measurement with MR images. Kaohsiung J Med Sci 1999;15(1):12-18.

31- Kiliç $\mathrm{N}$, Oktay $\mathrm{H}$. Effects of rapid maxillary expansion on nasal breathing and some nasorespiratory and breathing problems in growing children: a literature review. Int Pediat Otorhinolaryngol 2008;72:1595-1601.

32- Krakauer LH, Guilherme A. Relationship between mouth breathing and postural alterations of children: a descriptive analysis. Int J Orofacial Myology 2000; 26:13-23.

33- Kurt G, Altug-Ataç AT, Atac MS, Karasu HA. Changes in Nasopharyngeal Airway Following Orthopedic and Surgically Assisted Rapid Maxillary Expansion. J Craniofacial Surgery 2010;21(2):31217.

34- Lagrave're Mo, Major PW, Flores-Mir C. Long term skeletal changes with rapid maxillary expansion: a systematic review. Angle Orthod 2005;75:1046-52.

35- Langer MR, Itikawa CE, Valera FC, Matsumoto MA, Anselmo-Lima WT. Does rapid maxillary expansion increase nasopharyngeal space and improve nasal airway resistance? Int J Pediatr Otorhinolaryngol 2011;75(1):122-5. 
36- Lenza MG, Lenza MMO, Daltra M, Melsen B, Cattaneo PM. An analysis of different approaches to the assessment of upper airway morphology: a CBCT study. Orthod Craniof Res 2010;13:96-105.

37- Lessa FCR, Enoki C, Feres MFN, Valera FCP, Anselmo-Lima WT, Matsumoto MAN. Influência do padrão respiratório na morfologia craniofacial. Ver Bras Otorrinolaringol 2005; 71(2):156-60.

38- Linder-Aronson S. Adenoids: their effect on the mode of breathing and nasal airflow and their relationship to characteristics of the facial skeleton and the dentition. Acta Otolaryng Suppl 1970; 265:5-132.

39- Linder-Aronson S, Henrikson CO. Radiocephalometric analysis of anteroposterior nasopharyngeal dimensions in 6-to-12-year-old mouth breathers compared with nose breathers. J Otorhinolaryngol 1973;35:19-29.

40- Linder-Aronson S, Leighton BC. A longitudinal study of the development of the posterior nasopharyngeal wall between 3 and 16 years of age. Eur J Orthod 1983;5(1):47-58.

41- Lione R, Ballanti F, Franchi L, Baccenti T, Cozza P. Treatment and posttreatment skeletal effects of rapid maxillary expansion studied with low-dose computed tomography in growing subjects. Am J Orthod Dentofacial Orthop 2008; 134:89-92.

42- Lowe AA. Can we predict the success of dental appliance therapy for the treatment of obstructive sleep apnea based on anatomic considerations. Sleep 1993;16:93-95.

43- Lusvarghi L. Identificando o respirador bucal. Assoc Paul Cir Dent 1999; 53(4): 265-74.

44- Major MP, Flores-Mir C, Major PW. Assesment of lateral cephalometric diagnosis of adenoid hypertrophy and posterior airway obstruction: A systematic review. Am J Orthod Dentofacial Orthop 2006;130:700-8.

45- Matsumoto MAN, Itikawa CE, Valera FC, Faria G, Anselmo-Lima WT. Long-term effects of rapid maxillary expansion on nasal area and nasal airway resistance. Am J Rhinol Allergy 2010;24(2):161165.

46- Mattar SEM, Anselmo-Lima WT, Valera FCP, Matsumoto MAN. Skeletal and occlusal characteristics in mouth-breathing pre-school children. J Clin Pediatr Dent 2004; 28(4): 315-318.

47- McNamara JA Jr. Influence of respiratory pattern on craniofacial growth. Angle Orthod 1981;51:269-300. 
48- Monini S, Malagola C, Villa MP, Tripodi C, Tarentini S, Malagnino I, Marrone V, Lazzarino Al, Barbara M. Rapid maxillary expansion for the treatment of nasal obstruction in children younger than 12 years. Arch Otolaryngol Head Neck Surg 2009; 135(1):22-27.

49- Moore RN. Principles of dentofacial orthopedics. Semin Orthod 1997;3:212-21.

50- Moss ML. The primary role of functional matrices in facial growth. Am J Orthod 1969; 55(6):56677.

51- Pereira FC, Motonaga SM, Faria PM, Matsumoto MAN, Trawitzki IVV, Lima AS, Anselmo-Lima WT. Avaliação cefalométrica e miofuncional em respiradores bucais. Rev Bras Otorrinolaringol 2001; 67(1): 43-49.

52- Picchi F, Fiorelli G, Bolognini E, Piccini A. Otorhinological evaluations of patients undergoing rapid disjunction of the median palatine suture . Minerva Stomatologica 1990; 39:15-18.

53- Pirila-Parkkinen $\mathrm{K}$, Lopponen $\mathrm{H}$, Nieminen $\mathrm{P}$, Toloven U, Paakko E, Pirttiniemi P. Validity of upper airway assessment in children. Angle Orthod 2011; Published online: January 24.

54- Principato JJ. Upper airway obstruction and craniofacial morphology. Otolaryngol Head Neck Surg 1991;104(6):881-90.

55- Ramires T, Maia RA, Barone JR. Nasal cavity changes and the respiratory standard after maxillary expansion. Rev Bras Otorrinolaringol 2008;74(5):763-9.

56- Santos-Pinto CCM, Santos-Pinto PR, Ramalli EL, Santos-Pinto A, Raveli DB. Espaço nasofaringeano: avaliação pela telerradiografia. R Clin Ortodon Dental Press 2006;4(6):56-62.

57- Schwab RJ, Goldeberg NA. Upper airway assesment: radiographic and other imaging techniques. Otolaryngol Clin North Am 1998;31(6):931-68.

58- Songu M, Adibelli ZH, Tuncyurek O, Adibelli H. Age-Specific Size of the Upper Airway Structures in Children During Development. Annals of Otology. Rhinology \& Laryngology 2010;119(8):541-546.

59- Souza BB, Hennemann GV, Anselmo-Lima WT. Importance of nasal fiberoptic examination in the presence of a normal X-ray of the cavum. Int J Pediatric Otorhinolaryngol 2000;15(55):29-32.

60- Stuck BA, Köpke J, Maurer JT, Verse T, Kuciak G, Düber C, Hörmann K. Evaluating the upper airway with standardized magnetic resonance imaging. Laryngoscope 2002;112(3):552-8. 
61- Thomazinho A, Ferreira JTL, Stuani MBS, Matsumoto MAN. Ortodontia Preventiva e Interceptadora. In:Assed S. Odontopediatria, bases científicas para a prática clínica. São Paulo: Artes Médicas; 2005.p.879-992.

62- Valera FC, Travitzki IV, Mattar SE, Matsumoto MAN, Elias AM, Anselmo-Lima WT. Muscular, functional and orthodontic changes in preschool children with enlarged adenoids and tonsils. Int J Pediatr Otorhinolaryngol 2003;67(7):761-770.

63- Vig KWL. Nasal obstruction and facial growth: the strength of evidence for clinical assumptions. Am J Orthod Dentofacial Orthop 1998; 113:603-611.

64- Vig PS, Hall DJ. The inadequacy of cephalometric radiographs for airway assessment. Am J Orthod 1980;77:230-3.

65- Vilella BS, Vilella O, Koch H. Growth of the nasopharynx and adenoidal development in Brasilian subjects. Bras Oral Res 2006;20(1):70-7.

66- Vogler RC, Wippold FJ, Pilgram TK. Age-specific size of the normal adenoid pad on magnetic resonance imaging. Clin Otolaryngol Allied Sci 2000;25(5):392-95.

67- Warren DW, Spalding PM. Dentofacial morphology and breathing: a century of controversy. In: Melsen B. Current controversies in orthodontics. Quintessence Publishing; 1991.p.45-77.

68- Welch KC, Foster GD, Ritter CT, Waddwn TA, Arens R, Maislin G, Schwab RJ. A novel volumetric magnetic resonance imaging paradigm to study upper airway anatomy. Sleep 2002;25(5):530-40.

69- Yamada T, Tanne K, Miyamoto K, Yamauchi K. Influences of nasal respiratory obstruction on craniofacial growth in Young macaca fuscata monkeys. Am J Orthod Dentofacial Orthop 1997;111(1):38-43.

70- Ysunza A, Pamplona MC, Ortega JM, Prado H. Videofluoroscopy for evaluating adenoid hypertrophy in children. Int J Pediatr Otorhinolaryngol 2008;72(8):1159-65. 
ANEXOS 
ANEXO A - Aprovação pelo Comitê de Ética

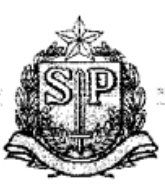

wwhherp.fme tusp.bF

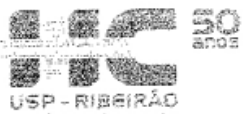

Ribeirāo Preto, 28 de março de 2007

Oficio $n^{\circ} 956 / 2007$

$\mathrm{CEP} / \mathrm{SPC}$

Prezada Professora,

O trabalho intitulado "AVALIAÇÃO DIMENSIONAL DÃ RAZĀO ADENÓTDE-NASOFARINGE (RAN) ATRAVES DA RESSONÂNCIA MAGNÉTICA E RINOMETRIA ACÚSTICA ANTES E APÓS A EXPANSÃo RÁPIDA DA MAXILÃ", foi analisado pelo Comitê de Ética em Pesquisa, em sua $243^{2}$ Reunião Ordinária realizada em 26/03/2007, e enquadrado na categoria: APROVADO, bem como o Termo de Consentimento Livre e Esclarecido, de acordo com o Processo HCRP n ${ }^{\circ}$ 13658/2006.

Atenciosamente.

PROF. DR. SÉRGIO PEREIRA DA CUNHA

Coordenador do Conaitê de Ética em

Pesquisa do HCRP e da FMRP-USP

Ilustríssima Senhora

PROF ${ }^{2}$ DR $^{2}$ WILMA TEREZINHA ANSELMO LIMA

MÁRCIO JOSÉ REIS (Orientando)

Depto. de Oftalmo, Otorrino e Cirurgia de Cabeça e Pescoço

Otorrinolaringologia 


\section{ANEXO B - Termo de Consentimento Livre e Esclarecido}

O seu filho(a) está sendo convidado a participar da pesquisa intitulada "Avaliação dimensional da razão nasofaringe (RAN) através da ressonância magnética e rinometria acústica antes e após expansão rápida da maxila" que será realizada no Centro do Respirador Bucal do Hospital das Clínicas da Faculdade de Medicina de Ribeirão Preto da Universidade de São Paulo e que tem como responsáveis a Profá. Drạ. Wilma T. AnselmoLima, a Profa. Dra. Mírian A. N. Matsumoto e os cirurgiões-dentistas Márcio José Reis e Fernanda W. M. Gallarreta. Informações sobre a pesquisa:

1- Objetivo e justificativa da pesquisa: a pesquisa tem como objetivo verificar se crianças que possuem alteração na mordida dentária (mordida cruzada) e dificuldade respiratória, apresentam melhora na sua respiração após o uso de um aparelho dentário que corrige a sua mordida. Com isso, a obstrução respiratória poderá ser diminuída evitando que se realize algum tipo de cirurgia para melhorar a respiração, além de se conseguir a correção da mordida dentária antes de todos os dentes permanentes estarem em boca.

2- Procedimentos que serão realizados:

- O médico otorrinolaringologista fará uma avaliação para verificar se a criança respira pela boca ou pelo nariz. Fará também exames em dois aparelhos: um deles, que serve para ver o tamanho da adenóide, poderá causar leve desconforto nasal, pois é colocada uma sonda flexível (cânula mole) dentro do nariz que estará com anestésico líquido (gotas); o outro que serve para saber a quantidade de ar que passa pelo nariz, não causará desconforto algum, pois a criança irá respirar normalmente em um copinho de vidro. Esse último será feito antes de colocar o aparelho nos dentes e depois de retirá-lo.

- O dentista irá verificar a posição dos dentes. Serão feitas moldagens das arcadas dentárias onde a criança morde em uma massa mole para copiar a forma dos dentes e que poderá causar náuseas. Serão realizadas 4 radiografias dos dentes e do rosto: uma de frente e outra de lado para ver os ossos do rosto; outra de frente, da região da boca, para ver todos os dentes e uma de dentro da boca (da arcada superior), para ver mais de perto os dentes que receberão o aparelho. Somente nessa última a criança poderá sentir náuseas e, as outras, não causarão desconforto algum, pois são feitas pelo lado de fora do rosto. Serão feitas, ainda, fotografias do rosto e dos dentes da criança.

- O dentista irá colocar um aparelho que será fixo nos dentes superiores que irá alargar o palato (céu da boca) para descruzar a mordida e não causará maiores desconfortos, a não ser durante a sua adaptação inicial. A permanência deste aparelho na boca será por volta de 06 meses e o dentista irá orientar o responsável pela criança de como realizar os ajustes e os cuidados necessários.

- Serão realizados dois exames de ressonância magnética (um antes de colocar o aparelho nos dentes e outro depois de retirá-lo) que serve para avaliar internamente o corpo da criança na região da cabeça (nariz, boca, adenóide). Nesse exame, a criança ficará deitada em uma maca que irá deslizar para dentro do aparelho que fará o exame, porém ele não irá tocar a criança. $O$ exame demora uns 15 minutos e a criança deve ficar sem se mexer durante esse tempo. O exame não faz mal à sua saúde e não causa nenhum desconforto, a não ser pelo tempo que deverá ficar sem se mexer.

3- Desconfortos e riscos possíveis: a criança não corre nenhum risco com a pesquisa. Se a criança sentir algum desconforto igual ou maior do que os citados acima durante a realização dos exames e não quiser mais fazê-los, ela não será obrigada, em hipótese alguma, a prosseguir na pesquisa. 
4- Forma de acompanhamento: os pesquisadores comprometem-se a prestar assistência integral à criança, garantindo tratamento para qualquer problema decorrente ou detectado durante o estudo, bem como o seu acompanhamento pelo tempo que for necessário (que varia conforme a criança).

5- Informações adicionais - direitos e condições asseguradas:

5-A) A garantia de receber esclarecimento de qualquer dúvida referente à pesquisa a ser realizada;

5-B) A liberdade de retirar o meu consentimento que permite a participação de meu filho(a) na pesquisa, a qualquer momento, sem sofrer penalizações;

5-C) A garantia de que será mantido sigilo sobre a identidade de meu filho(a) e que os dados obtidos na sua avaliação não terão qualquer relação nominal quando da publicação dos resultados da pesquisa;

5-D) O ressarcimento de eventuais despesas decorrentes da participação na pesquisa, tais como passagens, será promovido pelos pesquisadores responsáveis;

5-E) O ressarcimento de eventuais despesas, bem como a indenização, a título de cobertura material para a reparação de danos imediatos ou tardios, decorrentes da participação do menor na pesquisa, como por exemplo a confecção de novo aparelho dentário, será feito pelos pesquisadores responsáveis, não cabendo ao Hospital das Clínicas de Ribeirão Preto qualquer responsabilidade quanto aos referidos pagamentos.

5-F) Os pesquisadores tornarão públicos os resultados da pesquisa, sejam eles favoráveis ou não, mantendo o sigilo das crianças participantes, cumprindo as regulamentações da resolução 196/1996 do Conselho Nacional de Saúde.

\section{Endereço e telefone para contato em caso de dúvida:}

Dentista Márcio: Rua Cândido Junqueira, 205 - Centro - Itaú de Minas - Minas Gerais. Fone (35) 3536-1158 ou (35) 91996640.

Ou Centro do Respirador Bucal: Rua das Paineiras - Casa 20 - Campus USP - Ribeirão Preto SP. Fone (16) 3602-3363.

Baseado nas explicações que me foram prestadas sobre a pesquisa, eu, responsável pelo (a)

menor declaro que concordo

inteiramente com as informações e as condições que me foram apresentadas nesse termo de consentimento e que, livremente, manifesto minha vontade de meu filho (a) participar da referida pesquisa.

Ribeirão Preto, de de 